\title{
Plant Growth Promotion Driven by a Novel Caulobacter Strain
}

\author{
Dexian Luo, ${ }^{1,2}$ Sarah Langendries, ${ }^{1,2}$ Sonia Garcia Mendez, ${ }^{1,2,3}$ Joren De Ryck, ${ }^{1,2}$ Derui Liu, ${ }^{1,2}$ \\ Stien Beirinckx, ${ }^{1,2,4}$ Anne Willems, ${ }^{3}$ Eugenia Russinova, ${ }^{1,2}$ Jane Debode, ${ }^{4}$ and Sofie Goormachtig ${ }^{1,2,+}$ \\ ${ }^{1}$ Department of Plant Biotechnology and Bioinformatics, Ghent University, 9052 Ghent, Belgium \\ ${ }^{2}$ Center for Plant Systems Biology, VIB, 9052 Ghent, Belgium \\ ${ }^{3}$ Department of Biochemistry and Microbiology, Faculty of Sciences, Ghent University, 9000 Ghent, Belgium \\ ${ }^{4}$ Plant Sciences Unit, Flanders Research Institute for Agriculture, Fisheries and Food (ILVO), 9820 Merelbeke, Belgium
}

Accepted 28 March 2019.

\begin{abstract}
Soil microbial communities hold great potential for sustainable and ecologically compatible agriculture. Although numerous plant-beneficial bacterial strains from a wide range of taxonomic groups have been reported, very little evidence is available on the plant-beneficial role of bacteria from the genus Caulobacter. Here, the mode of action of a Caulobacter strain, designated RHG1, which had originally been identified through a microbial screen for plant growth-promoting (PGP) bacteria in maize (Zea mays), is investigated in Arabidopsis thaliana. RHG1 colonized both roots and shoots of Arabidopsis, promoted lateral root formation in the root, and increased leaf number and leaf size in the shoot. The genome of RHG1 was sequenced and was utilized to look for PGP factors. Our data revealed that the bacterial production of nitric oxide, auxins, cytokinins, or 1-aminocyclopropane-1-carboxylate deaminase as PGP factors could be excluded. However, the analysis of brassinosteroid mutants suggests that an unknown PGP mechanism is involved that impinges directly or indirectly on the pathway of this growth hormone.
\end{abstract}

Keywords: genetics and gene regulation, genomics, microscopy and imaging, molecular signaling, rhizosphere and phyllosphere ecology.

Plant roots are associated with a wide variety of bacteria from different taxonomic groups, many of which can enhance plant growth or stress tolerance via diverse mechanisms, including hormone modulation (Tsukanova et al. 2017), nutrient uptake enhancement (Richardson et al. 2009; Van Deynze et al. 2018), or disease suppression (Berendsen et al. 2018; Kwak et al. 2018). These bacteria, referred to as plant-growth-promoting (PGP) rhizobacteria (PGPR) (Vacheron et al. 2013), have received increasing attention, because they provide a sustainable and ecological solution for the agricultural challenges we are facing (Gouda et al. 2018; Toju et al. 2018).

${ }^{\dagger}$ Corresponding author: S. Goormachtig; sogoo@psb.vib-ugent.be

Funding: This work was supported by a grant from the Research Foundation-Flanders (project number G015118N) to A. Willems and S. Goormachtig.

*The $\boldsymbol{e}$-Xtra logo stands for "electronic extra" and indicates that seven supplementary figures are published online.

The author(s) declare no conflict of interest.

(c) 2019 The American Phytopathological Society
Because phytohormones control plant growth and its interaction with the environment, PGPR have been proposed to affect the plant hormonal landscape for their establishment in or around the plant roots and for growth stimulation. Indeed, PGPR have been shown to be able to generate phytohormones, such as auxins and cytokinins, or enzymes and compounds that interfere with the endogenous phytohormone production or with signaling pathways. For instance, Azospirillum brasilense $\mathrm{Sp} 245$ is an auxin-producing PGPR strain that promotes lateral root formation and enhances the expression of auxin-responsive genes in Arabidopsis thaliana. Both effects are impaired in the auxin biosynthesis mutant strain FAJ0009 (Spaepen et al. 2014). Also, cytokinins that play an essential role in cell division and influence many aspects of plant growth and development (Schaller et al. 2014) have been shown to be produced by PGPR such as species of Bacillus (Liu et al. 2013) and Pseudomonas (Pallai et al. 2012). Additionally, 1-aminocyclopropane-1-carboxylate (ACC) deaminase-producing PGPR such as Pseudomonas putida UW4 (Hontzeas et al. 2004) modulate the plant ethylene levels by degrading the plant-generated ACC, thereby mitigating the plant-growth-inhibitory effects of ethylene (Glick 2014). Conversely, PGPR have been found to regulate plant hormonal homeostasis without production of the respective hormones. For example, the volatile indole emitted by the rhizobacterium Proteus vulgaris JBLS202 enhances Arabidopsis growth through an interplay between the auxin, cytokinin, and brassinosteroid pathways (Bhattacharyya et al. 2015). Another example is the Pseudomonas model strain Pseudomonas simiae WCS417, of which the auxin-producing ability had not been detected by either colorimetric assays in bacterial cultures or genome analysis (Berendsen et al. 2015; Zamioudis et al. 2013). However, both the induction of the PGP effect and expression of the induced systemic resistance (ISR) markers by this rhizobacterium depend on auxin signaling in Arabidopsis (Stringlis et al. 2018; Zamioudis et al. 2013). The early transcriptional response of Arabidopsis roots to this bacterium has a strong auxin signature (Stringlis et al. 2018) but the bacterial factors responsible for this response remain unknown.

Nitric oxide (NO), another key signaling molecule involved in diverse plant developmental processes and stress responses, is generated by bacteria and proposed to be a phytostimulating signal (Sami et al. 2018). In bacteria, NO production is mainly mediated by two types of nitrite reductase (Nir): the haem-cytochrome $c d 1$ type encoded by nirS genes and the copper-containing type encoded by nirK genes (Zumft 1997). The Azospirillum brasilense strain Sp245 possesses two copies of the nirK gene (Pothier et al. 
2008) and this strain generates NO on tomato (Solanum lycopersicum) roots (Creus et al. 2005). The Azospirillum-induced lateral root formation was blocked by the NO scavenger 2(4-carboxyphenyl)-4,4,5,5-tetramethylimidazoline-1-oxyl-3-oxide, hinting at the involvement of NO in this process (Creus et al. 2005). Correspondingly, the P. kilonensis F113-mediated modification of the root system architecture was significantly impaired in the nirS gene deletion mutant (Vacheron et al. 2018).

Next-generation sequencing analysis revealed that many more bacterial genera live in root endospheres and rhizospheres than previously anticipated (Bai et al. 2015; Fitzpatrick et al. 2018; Walters et al. 2018). Currently, for many of these genera, of which Caulobacter is one, no clues are supplied as to whether they contribute to plant growth or health. The Caulobacter genus has been frequently found in endospheres or rhizopheres of various plants grown in different soils (Bai et al. 2015; Fitzpatrick et al. 2018; Walters et al. 2018). Interestingly, the Caulobacter genus acts as one of the microbial "hubs" that are strongly interconnected and have a central impact on the leaf microbiome of Arabidopsis (Agler et al. 2016). Additionally, different Caulobacter strains have been isolated from diverse plants or rhizosphere soil, including Caulobacter rhizosphaerae $7 \mathrm{~F} 14^{\mathrm{T}}$ from the rhizosphere soil of watermelon (Citrullus lanatus) (Sun et al. 2017), Caulobacter sp. HGR25 from horse gram (Macrotyloma uniflorum (Lam.) Verdc.) (Edulamudi et al. 2011), and Caulobacter hibisci
THG-AG3. $4^{\mathrm{T}}$ from the rhizosphere of Mugunghwa flower $(\mathrm{Hi}$ biscus syriacus L.) (Moya et al. 2017), indicating that the association between Caulobacter spp. and plants is ubiquitous in natural environments. However, little is known about the beneficial role of the Caulobacter members on plants.

Here, we examine the Caulobacter sp. strain RHG1 that had been identified through a microbial screen for PGP endophytic bacteria in maize (Zea mays). The root colonization of the green fluorescent protein (GFP)-labeled strain was thoroughly analyzed in Arabidopsis by confocal microscopy. The PGP effect was investigated by an in-depth growth analysis, the potential PGP traits in the RHG1 genome were examined, and, concomitantly, the impact on colonized plants was studied on Arabidopsis mutants and marker lines affected in or representative for diverse hormone pathways, respectively.

\section{RESULTS}

RHG1 promotes plant growth in Arabidopsis.

The PGP effect of Caulobacter sp. RHG1 in Arabidopsis was studied in an in vitro system, in which Arabidopsis seeds were inoculated with RHG1. Eighteen days after initiation of germination (DAIG), the fresh weights of shoots and roots had significantly increased after the bacterial inoculation (Fig. 1A and B).
A

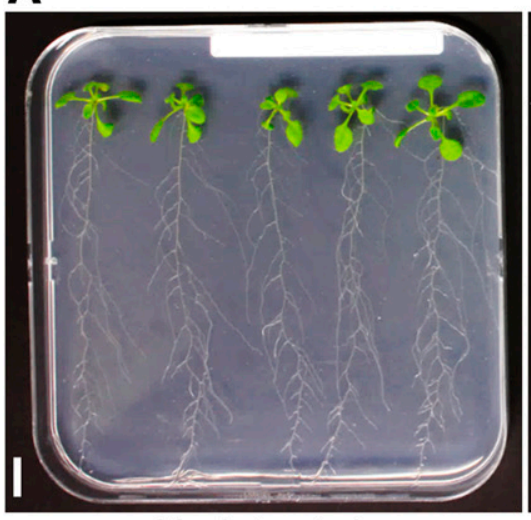

Mock-treated

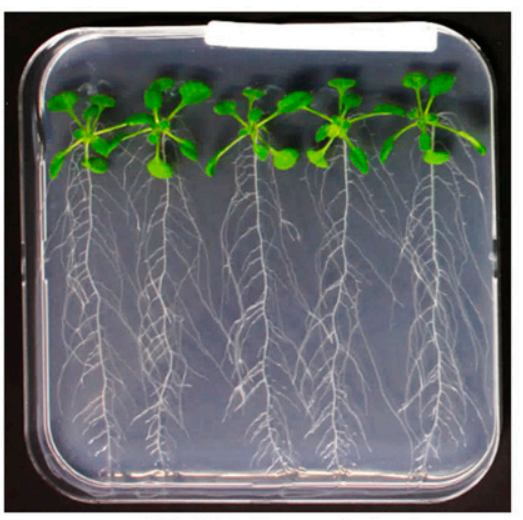

RHG1-inoculated

B

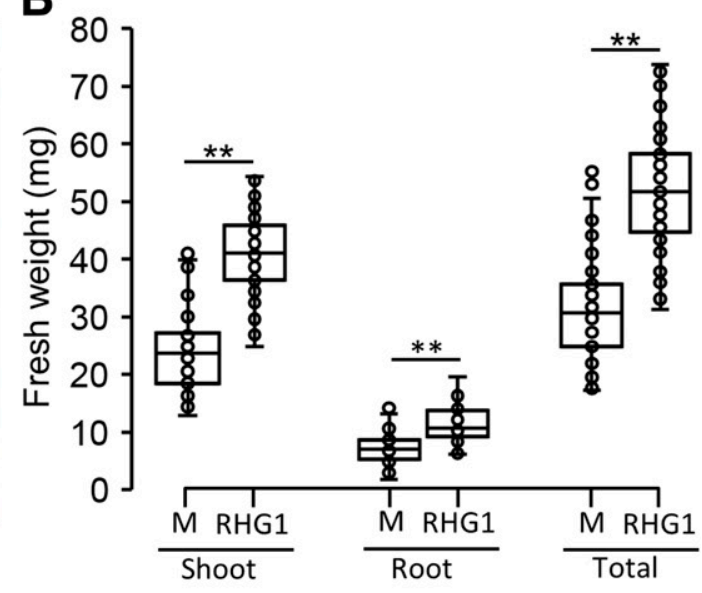

E

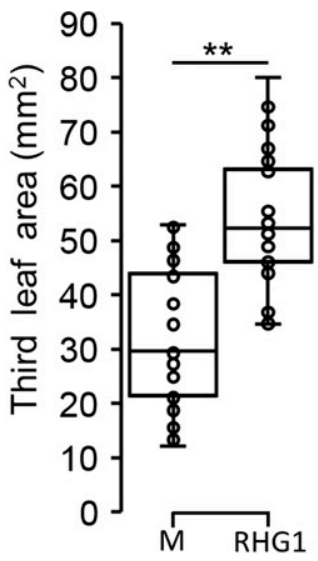

D

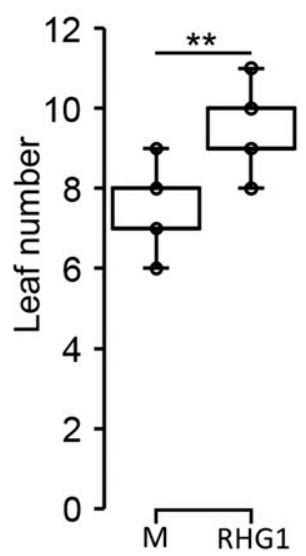

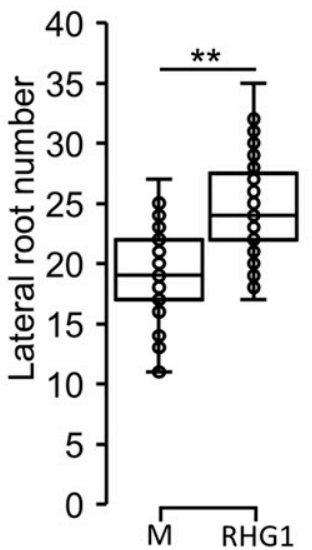

$\mathbf{F}$

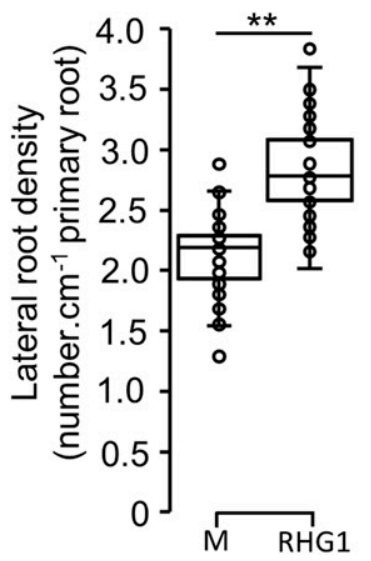

G

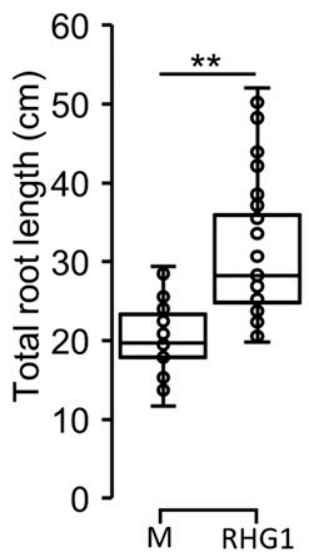

Fig. 1. Plant growth promotion effect of RHG1 in Arabidopsis. Seeds were mock treated or inoculated with RHG1 in an in vitro system and the different growth parameters were determined 14 or 18 days after initiation of germination (DAIG). A, Representative photographs of mock-treated or RHG1-inoculated Arabidopsis seedlings at 18 DAIG. Bar $=1 \mathrm{~cm}$. Effects of RHG1 on different parameters of Arabidopsis plant growth: B, fresh weight; C, third leaf area; and D, leaf number were determined at 18 DAIG and E, lateral root number; F, lateral root density; and G, total root length at 14 DAIG. Plot graphs are based on three biological replicates ( $n \geq 12$ for each treatment). Asterisks indicate significant differences between mock and inoculated plants ( $* *$ indicates $P<0.01$, Student's $t$ test). $\mathrm{M}=$ mock-treated and RHG1 = RHG1-inoculated. 
Because growth is a complex trait that integrates many different factors, among which are control of organ initiation, cell division, and cell expansion (Kalve et al. 2014), we examined how these factors could be influenced by the RHG1 inoculation. In the shoot, increase in both leaf size and total leaf number contributed to the enhancement of the shoot biomass of the inoculated plants (Fig. 1C and D). Furthermore, cellular analysis of the leaf epidermis revealed that the number of pavement cells, guard cells, and, accordingly, total epidermal cells was higher in plants inoculated with RHG1 than that of mock-treated plants (Fig. 2A to C). Interestingly, the pavement cells in the leaves of RHG1-inoculated plants were significantly smaller than those of mock-treated plants (Fig. 2D), suggesting that the enlargement in leaf size by RHG1 results from an increase in cell number instead of cell area. To assess the influence of RHG1 on root growth, we determined primary root length, lateral root number, lateral root density, and total root length at 14 DAIG. RHG1 inoculation had a variable effect on the primary root length. The RHG1-inoculated plants had significantly shorter primary roots than the mock-treated roots in some of the experiments but this phenotype was not stable in every experiment (Supplementary Fig. S1). However, in all of the experiments, the impact of the RHG1 inoculation on the lateral root number, the lateral root density, and the total root length was consistent and positive (Fig. 1E to G), contributing to the increase in root biomass of the inoculated plants.

To evaluate whether the PGP effect of RHG1 was caused by the bacterial production of $\mathrm{CO}_{2}$ or other easily diffusible volatile compounds, we used a cocultivation setup and tested whether the mock-treated plants grown next to the RHG1inoculated plants in the same plate could benefit from the bacteria. In this setup, the fresh weight and lateral root number of the RHG1-inoculated plants were significantly higher than those of the mock-treated plants but the increase in fresh weight and lateral root number by RHG1 in the cocultivation setup was not significantly different from that in plants grown in the setup in which the RHG1-inoculated and mock-treated plants were grown in different plates (Supplementary Fig. S2). This result

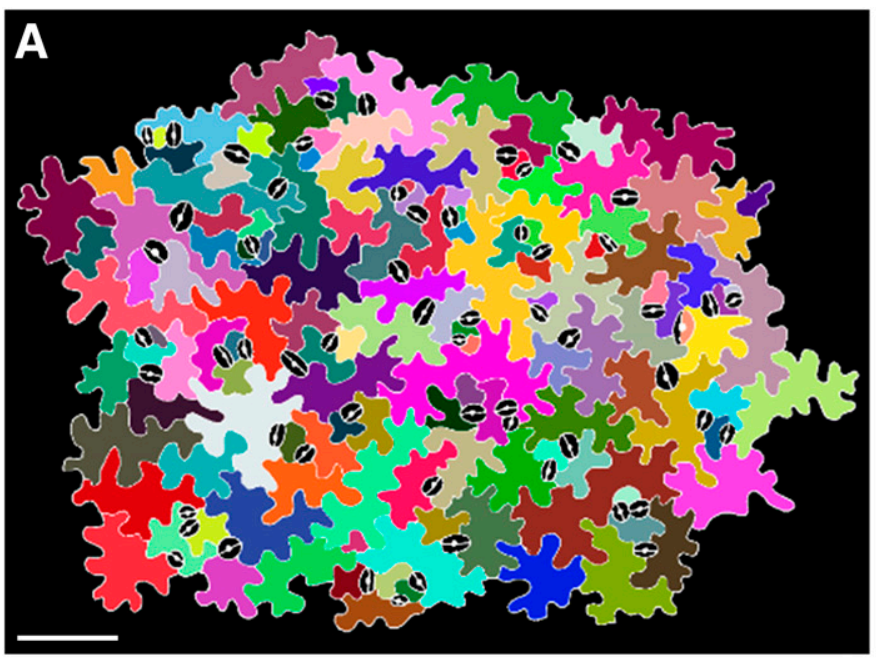

C

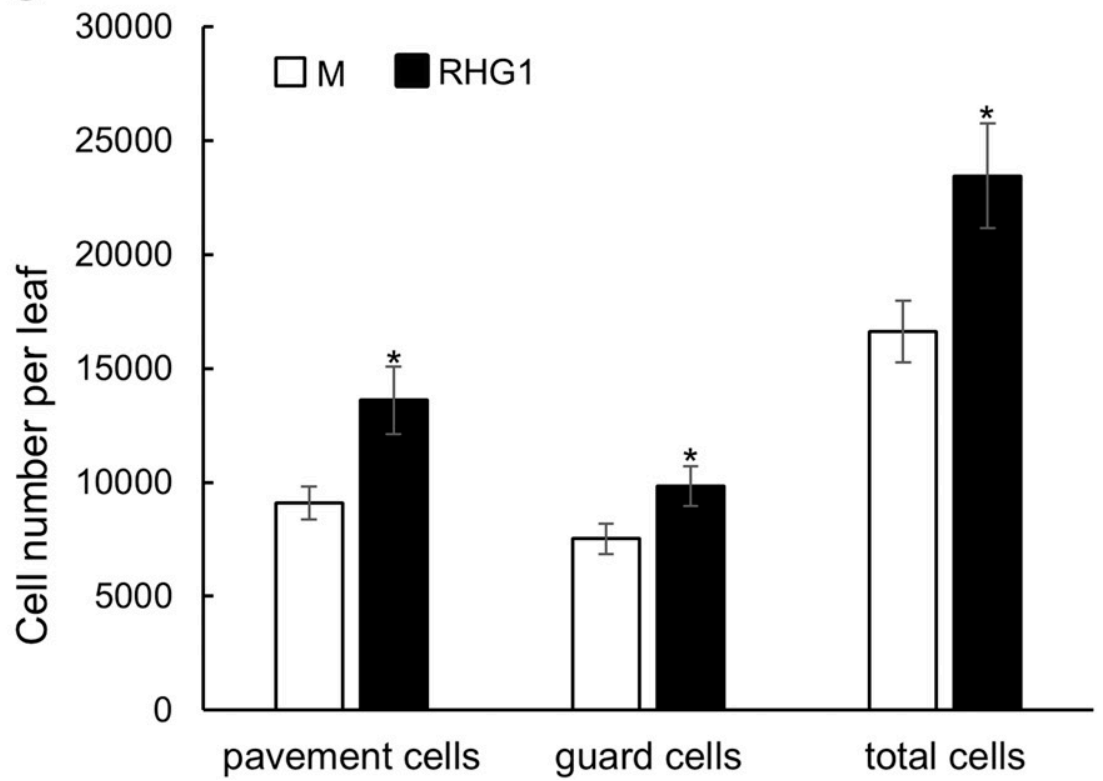

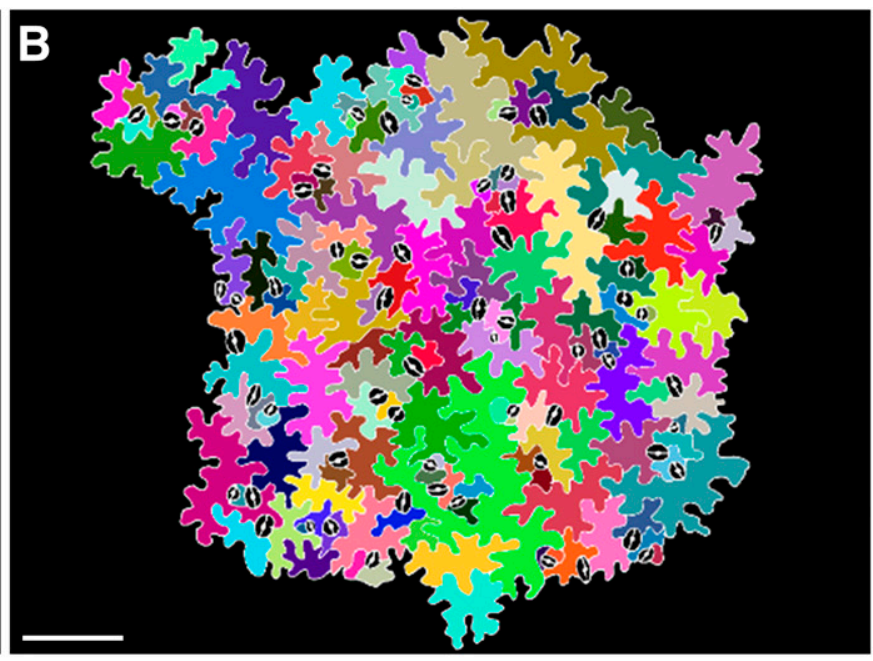

D

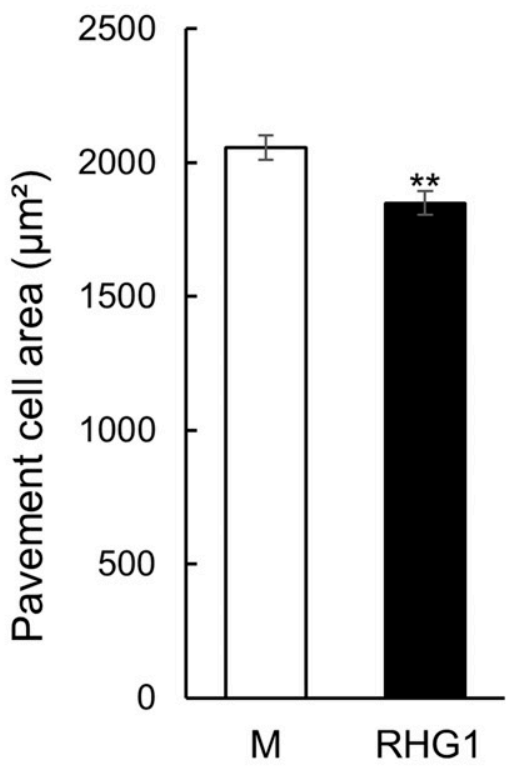

Fig. 2. Increased cell number and reduced cell area in leaves by RHG1 inoculation. Representative pictures of abaxial epidermal cells in leaves of A, mocktreated and B, RHG1-inoculated plants. Bar $=100 \mu \mathrm{m}$. C, Average number of pavement cells, guard cells, and total cells per leaf and D, average pavement cell area. Mean \pm standard error. Graphs are based on three biological replicates with six leaves for each treatment per replicate. Asterisks indicate significant differences between mock and inoculated plants (** and * indicate $P<0.01$ and 0.05 , respectively; Student's $t$ test). M mock-treated and RHG1 $=$ RHG1inoculated. 
suggested that the PGP effect of RHG1 is not caused by $\mathrm{CO}_{2}$ or other easily diffusible volatile compounds.

\section{Colonization pattern of RHG1 in Arabidopsis.}

Next, we investigated whether RHG1 could enter the plant and live as an endophyte. The RHG1 colonization on Arabidopsis shoots and roots was determined by counting CFU per milligram of fresh weight (FW). At 14 DAIG, the overall count was in the range of $10^{6}$ to $10^{7}$ (average $8.81 \times 10^{6}$ ) or $10^{6}$ to $10^{8}$ (average $9.51 \times 10^{7}$ ) CFU/mg FW in the shoots or roots, respectively. To examine the colonization pattern of RHG1 microscopically, we labeled RHG1 with GFP by means of the mini-Tn5-based transposon delivery system (Tombolini et al. 1997), which allowed the insertion of the GFP marker driven by a constitutive PpsbA promoter into the chromosome of the bacteria. The resulting strain is referred to as RHG1::GFP hereafter. The shoot and root weights of plants inoculated with the RHG1::GFP strain were higher than those of the mocktreated control but the fresh weights of the RHG1::GFPinoculated and the RHG1-inoculated plants did not differ significantly (Supplementary Fig. S3), indicating that the insertion of the GFP marker into the genome did not disturb the interaction of the bacteria with the plants. Then, we analyzed the colonization pattern of RHG1::GFP by using confocal microscopy. At 14 DAIG, colonization of RHG1::GFP was observed on the root and leaf surfaces (Fig. 3A to E). Additionally, at sites where lateral roots emerged, the GFP signal was visible between the plant cells (Fig. 3E). To improve the in-depth imaging within roots, we employed the ClearSee protocol (Kurihara et al. 2015) that fixes and clears the inoculated root with a mounting medium with a high refractive index that allows high light penetration. The microscopic observation of the cleared tissues confirmed the colonization by RHG1::GFP on the root surface and the intercellular positions at lateral root emergence (Fig. 3F to $\mathrm{H}$ ), as seen via imaging of live specimens. Hence, RHG1 colonizes the root and leaf surfaces and enters the root at the lateral root bases but without signs of colonization of the vascular tissues.

\section{Genome and encoded functions of RHG1.}

To test whether signs for root colonization and PGP traits could be found, we sequenced the RHG1 genome. The draft genome sequence was assembled with the Shovill platform. The total length of the RHG1 assembly was approximately 5.6 million bp, with an average $\mathrm{G}+\mathrm{C}$ content of $67.55 \%$. This assembly was made up of 19 contigs, of which the largest is $861,938 \mathrm{bp}$ and the smallest $1,000 \mathrm{bp}$. The N50 value is $643,962 \mathrm{bp}$. The draft genome of RHG1 presented 5174 coding sequences, 63 transfer RNA genes, and 4 ribosomal RNA (rRNA) genes.

Additionally, the RHG1 strain was subjected to a phylogenomic analysis by comparing its genome against that of 38 known strains of the genus Caulobacter and with three outgroups of the genus Brevundimonas as close relatives. The bcgTree software was utilized that evaluated 107 essential core genes found in most bacteria (mostly housekeeping and ribosomal proteins) (Ankenbrand and Keller 2016). Interestingly, RHG1 occurred in a clade with 15 other Caulobacter strains, including C. vibrioides (OR37, CB13b1a, CB2 ${ }^{\mathrm{T}}$ [formerly known as the type strain of $C$. crescentus], DSM $9893^{\mathrm{T}}, \mathrm{CB} 15$, NA1000, and CB1), C. segnis (ATCC $21756^{\mathrm{T}}$ ), and some unspecified strains (Caulobacter sp. FWC2, Caulobacter sp. OV484, Caulobacter sp. Root342, Caulobacter sp. Root343, Caulobacter sp. X, Caulobacter sp. BP25, and Caulobacter sp. FWC26) (Supplementary Fig. S4). Remarkably, RHG1 is the most peripheral in the group, clearly separated from all the other strains in this clade, suggesting that RHG1 is evolutionarily distant when compared with other known Caulobacter spp. To support these results, the similarity between RHG1 and the other strains was calculated by means of OrthoANIu (Yoon et al. 2017). The obtained values ranged from 72.02 to $84.58 \%$ average nucleotide identity (ANI), with the highest values with Caulobacter sp. OV484 (84.58\% ANI), Caulobacter sp. Root342 (84.57\% ANI), Caulobacter sp. Root343 (84.57\% ANI), and Caulobacter sp. FWC2 (84.36\% ANI). These results are consistent with the outcome of the bcgTree software, corroborating the observed evolutionary distance. Because the genome sequences are not available for all 11 validly named Caulobacter spp., the 16S rRNA genes available for all the type strains were used to construct a maximum-likelihood tree (Supplementary Fig. S5). In agreement with the results obtained in the phylogenomic analysis, RHG1 formed a separate lineage and its closest neighbors were C. vibrioides and C. segnis.

Special attention was given to the occurrence of genes linked to endophytic lifestyles (Mitter et al. 2013) and PGP functions (Lemanceau et al. 2017) (Table 1). Plant polymerdegrading enzymes (polysaccharide lyase and glycosyl hydrolases families) and reactive oxygen species-detoxifying enzymes (catalase, peroxidase, and superoxide dismutase), which have been proposed to be important for endophytic lifestyles (Mitter et al. 2013), were found as well as genes involved in flagellar motility and chemotaxis, which are key during the rhizosphere colonization. No genes occurred involved in ACC degradation (ACC deaminase) or auxin (such as tryptophan monooxygenase and indole-3-pyruvate decarboxylase), cytokinin (isopentenyl transferase), gibberellin (gibberellin 20-oxidase), or abscisic acid (isopentenylpyrophosphate isomerase and farnesyl diphosphate synthase) biosynthesis, or genes involved in biocontrol activity (2,4-diacetylphloroglucinol biosynthesis and cyanhidric acid biosynthesis) or in biofertilization (phosphate solubilization and nitrogen fixation). Interestingly, a NirK protein, as well as other proteins involved in the denitrification process (NO reductase and NO-responding transcriptional activator NnrR) were predicted from the genome of RHG1.

\section{The nitrite reductase gene nirK of RHG1 is not crucial for its PGP trait.}

The nirK gene present in the genome of RHG1 shows high similarity to known nirK genes from other bacteria, including A. brasilense Sp245 (Supplementary Fig. S6), which has been proposed to induce lateral root formation in tomato by the generation of NO (Creus et al. 2005; Pothier et al. 2008). This hypothesis raised the question whether the nirK gene-mediated NO production by RHG1 would contribute to its PGP function. Therefore, we tested whether deletion of nirK from the RHG1 genome might reduce the PGP effect of the bacterium. The nirK deletion mutant, referred to as RHG1 $\Delta$ nirK hereafter, was constructed through a recombination-based method (see Materials and Methods). The impact was analyzed of the RHG1 $\Delta n i r K$ inoculation on lateral root number, lateral root density, and shoot and root weights of Arabidopsis. In comparison with the wild-type RHG1 strain, the plant growth parameters did not differ significantly (Fig. 4), suggesting that the nirK gene is not essential for the PGP function of RHG1.

\section{Plant auxin, cytokinin, and ethylene signaling pathways are upregulated upon RHG1 inoculation, but probably not essential for the PGP effect.}

Bacterial production of auxins, cytokinins, or ACC deaminase is frequently found to be a PGP trait in PGPR. Pathways or genes responsible for the generation of these compounds did not occur in the draft RHG1 genome. However, 
these pathways can also be modulated by currently unknown bacterial compounds or enzymes. To rule out this hypothesis, we examined the responses of the plant hormone reporter lines to the RHG1 inoculation and assessed the PGP effect on the corresponding hormone mutant and transgenic lines. The expression of the auxin-responsive reporter DR5: $\beta$-glucuronidase (GUS) (Ulmasov et al. 1997) was upregulated by RHG1 in the shoot but not in the root (Fig. 5A). Consistently, the expression of DR5:GFP was not significantly altered by the bacteria in the root (Fig. 5A). The auxin mutant and transgenic lines yuccalD, 35S:iaaL, and tirlafb2/3 were tested for RHG1-mediated PGP effect. yuccalD is an indole-3-acetic acid (IAA)-overproducing gain-of-function mutant (Zhao et al. 2001); the 35S:iaaL line overexpresses the bacterial IAA lysine synthase that inactivates IAA (Jensen et al. 1998); tirlafb2/3 is an auxin signaling mutant deficient in the auxin receptors TIR1, AFB2, and AFB3
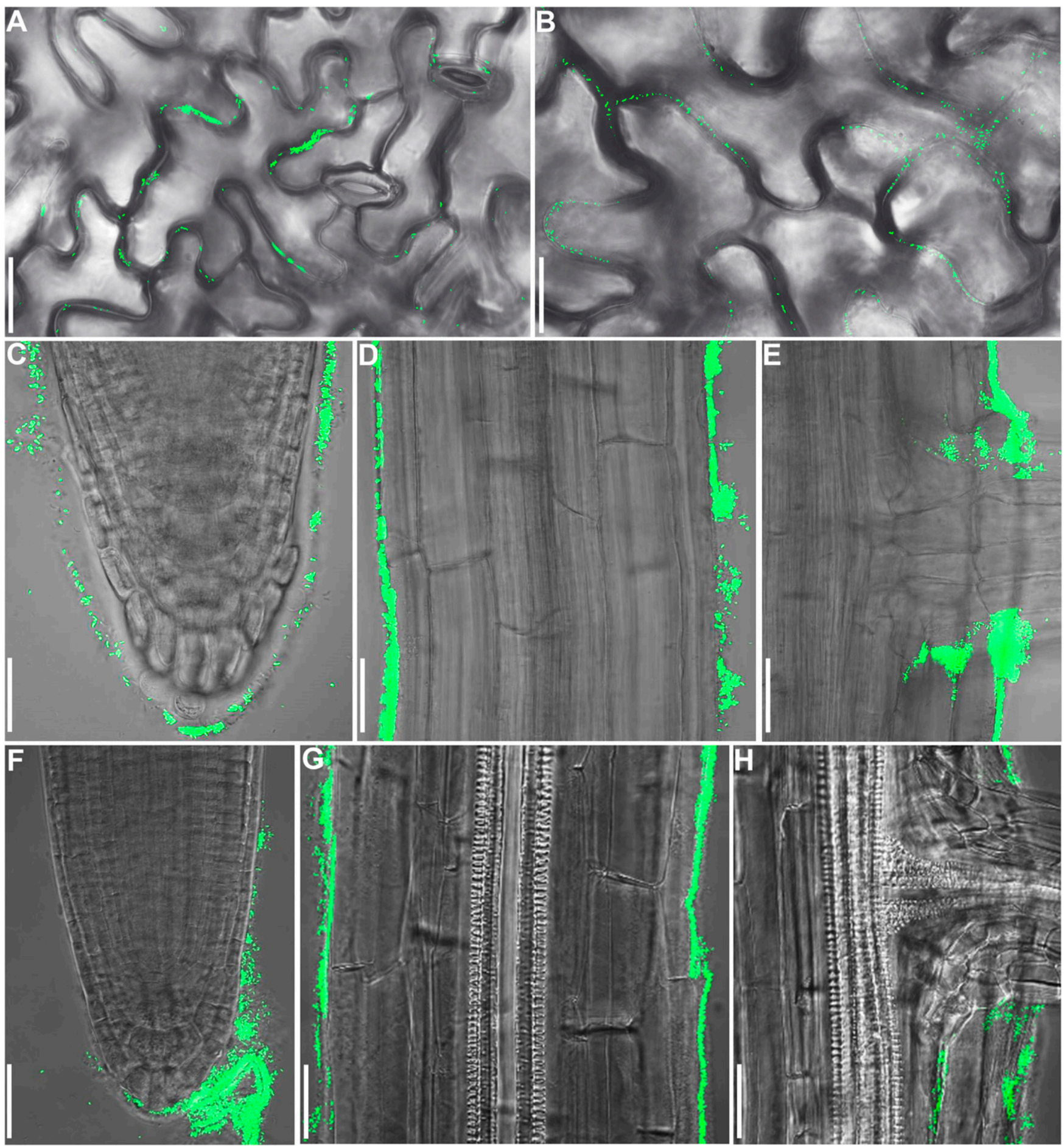

Fig. 3. RHG1::green fluorescent protein (GFP) colonization of Arabidopsis. Confocal microscopic images of Arabidopsis colonized by strain RHG1::GFP Seeds were inoculated with RHG1::GFP. At 14 days after initiation of germination (DAIG), plants were directly subjected to confocal microscopic imaging (A to E) or cleared in ClearSee buffer for 2 weeks followed by confocal microscopic imaging (F to H). RHG1::GFP colonization on the surface of the A, abaxial side and $\mathbf{B}$, adaxial side of the leaf. RHG1::GFP colonization of the root at $\mathbf{C}$ and $\mathbf{F}$, the root tip; $\mathbf{D}$ and $\mathbf{G}$, mature zone; and $\mathbf{E}$ and $\mathbf{H}$, lateral root emergence. $\mathrm{Bar}=30 \mu \mathrm{m}$. 
(Dharmasiri et al. 2005). Total fresh weight of the plant, instead of separate shoot or root weights, was measured, because the weights of 35 S:iaaL and tirlafb2/3 root were smaller than the minimum range of the analytic balance. The increase in plant fresh weight caused by RHG1 was not reduced in any of the auxin mutants or transgenic lines when compared with the Arabidopsis wild-type Columbia-0 (Col-0) (Fig. 5B).

Similarly, the cytokinin-responsive reporter $p A R R 5: G U S$ (D'Agostino et al. 2000) was slightly upregulated by the bacteria in the shoot but not in the root (Fig. 6A). However, RHG1

Table 1. Presence of traits in the RHG1 genome related to plant growth promotion and endophytic lifestyle ${ }^{\mathrm{a}}$

\begin{tabular}{|c|c|c|c|c|c|}
\hline Category & Function & Gene & NODE & Start & End \\
\hline \multirow[t]{5}{*}{ Polymer-degrading enzymes } & Polysaccharide lyase family & 1 & 3 & 33,609 & 34,985 \\
\hline & Glycosyl hydrolases family & 1 & 1 & 81,572 & 82,603 \\
\hline & $\ldots$ & 1 & 1 & 121,418 & 123,883 \\
\hline & $\ldots$ & 1 & 5 & 382,168 & 384,420 \\
\hline & $\ldots$ & 1 & 9 & 47,554 & 50,001 \\
\hline \multirow[t]{8}{*}{ ROS-detoxifying enzymes } & Catalase & 1 & 2 & 349,951 & 352,044 \\
\hline & $\ldots$ & 1 & 5 & 68,245 & 69,693 \\
\hline & $\ldots$ & 1 & 10 & 164,194 & 165,648 \\
\hline & Peroxidase & 1 & 3 & 343,660 & 344,496 \\
\hline & $\ldots$ & 1 & 5 & 71,385 & 72,365 \\
\hline & $\ldots$ & 1 & 7 & 92,478 & 92,939 \\
\hline & Superoxide dismutase & 1 & 3 & 657,325 & 657,942 \\
\hline & $\ldots$ & 1 & 11 & 146,429 & 146,953 \\
\hline \multirow[t]{8}{*}{ Motility and chemotaxis } & Flagellar motility & 8 & 1 & 133,513 & 139,962 \\
\hline & $\ldots$ & 3 & 1 & 444,115 & 446,245 \\
\hline & $\ldots$ & 5 & 1 & 664,047 & 665,673 \\
\hline & $\ldots$ & 11 & 2 & 72,375 & 80,644 \\
\hline & $\ldots$ & 13 & 8 & 209,728 & 225,726 \\
\hline & Chemotaxis & 6 & 2 & 130,282 & 137,690 \\
\hline & $\ldots$ & 7 & 5 & 297,807 & 304,828 \\
\hline & $\ldots$ & 14 & 5 & 390,511 & 405,982 \\
\hline \multirow[t]{2}{*}{ Biofertilization } & Phosphate solubilization & - & - & - & - \\
\hline & Nitrogen fixation & - & - & - & - \\
\hline \multirow{2}{*}{ Biocontrol } & 2,4-Diacetylphloroglucinol biosynthesis & - & - & - & - \\
\hline & Cyanhidric acid biosynthesis & - & - & - & - \\
\hline \multirow[t]{5}{*}{ Denitrification } & Copper-containing nitrite reductase & 1 & 2 & 236,063 & 237,094 \\
\hline & Nitrite reductase $[\mathrm{NAD}(\mathrm{P}) \mathrm{H}]$ small and large subunit & 2 & 2 & 114,040 & 116,852 \\
\hline & NO reductase & 1 & 1 & 281,420 & 283,696 \\
\hline & $\ldots$ & 1 & 2 & 230,222 & 232,495 \\
\hline & NO-responding transcriptional activator NnrR & 1 & 2 & 234,107 & 234,802 \\
\hline \multirow[t]{5}{*}{ Phytohormone modulation } & ACC deaminase & - & - & - & - \\
\hline & Indole-3-acetic acid biosynthesis & - & - & - & - \\
\hline & Gibberellin biosynthesis & - & - & - & - \\
\hline & Cytokinin biosynthesis & - & - & - & - \\
\hline & Abscisic acid biosynthesis & - & - & - & - \\
\hline
\end{tabular}

${ }^{a} \mathrm{ROS}=$ reactive oxygen species, $\mathrm{NO}=$ nitric oxide, $\mathrm{ACC}=1$-aminocyclopropane-1-carboxylate, and - indicates no gene found for the category.
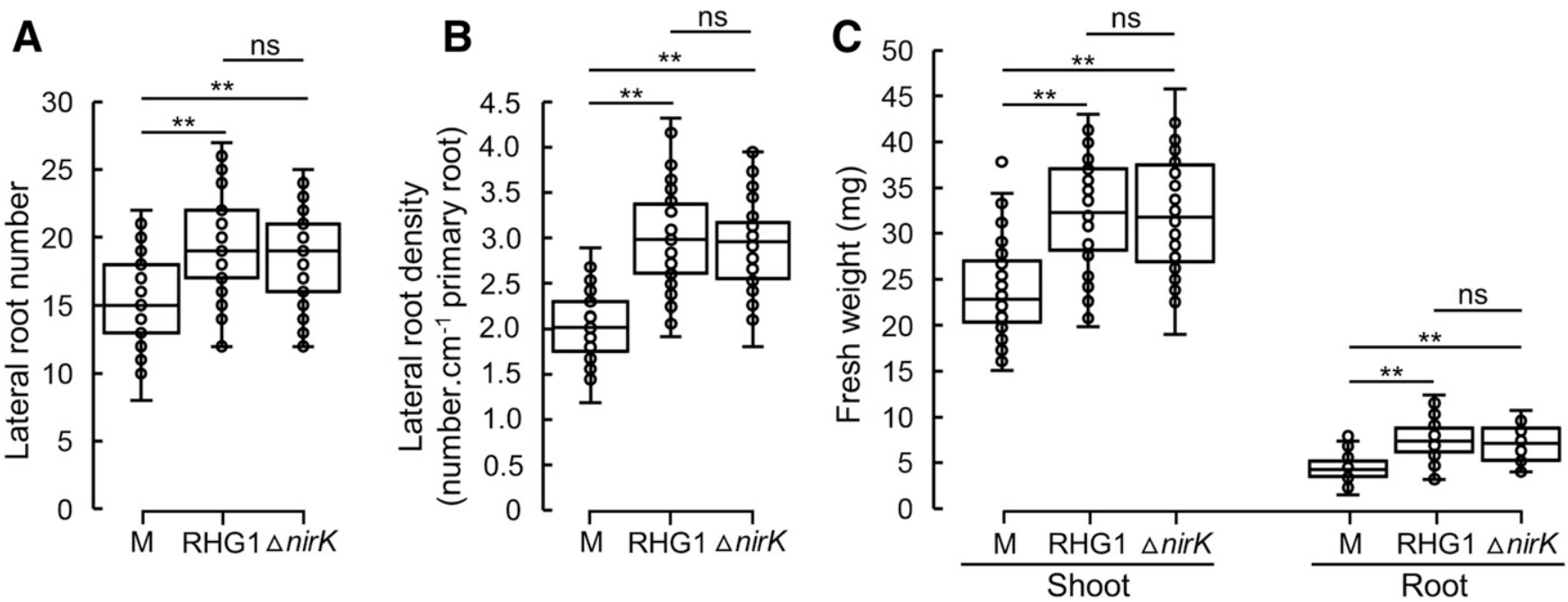

Fig. 4. Effects of RHG1 and RHG1 $\Delta$ nirK inoculation on different parameters of the Arabidopsis plant growth. Seeds were mock treated (M) or inoculated with the wild-type RHG1 strain (RHG1) or the RHG1 $\Delta$ nirK mutant strain ( $\Delta$ nirK). A and B, Lateral root number and lateral root density determined 14 days after initiation of germination (DAIG). C, Fresh weights of shoots and roots determined at 18 DAIG. Data of three independent biological repeats were combined $(n \geq 15)$. Asterisks indicate significant differences between different treatments (** indicates $P<0.01$ and ns indicates $P>0.05$; Student's $t$ test). 
significantly promoted growth in terms of fresh root and shoot weights in the ahk2/3/4 triple mutant, which is impaired in cytokinin signaling due to mutations in the cytokinin receptors AHK2, AHK3, and AHK4 (Nishimura et al. 2004) (Fig. 6B).

The expression of the ethylene-responsive marker $p E B S$ : GUS (Stepanova et al. 2007) was upregulated both in the shoots and roots of the RHG1-inoculated plants when compared with the mock-treated control (Fig. 6C). Hence, we also tested whether the ethylene signaling pathway is required for the PGP effect. In the ethylene-insensitive mutant ein2-5, which is deficient in ethylene signal transduction due to a null mutation in the ETHYLENE INSENSITIVE2 (EIN2) gene (Wang et al. 2007), the RHG1-mediated increase in plant weight was not attenuated when compared with the Arabidopsis wild-type Col0 (Fig. 6D). Collectively, although the expression of the markers responsive to auxin, cytokinin, and ethylene was slightly upregulated upon RHG1 inoculation, none of the corresponding mutants or transgenic lines had a reduced PGP effect, indicating that these hormones do not play a main role in the observed PGP effect.

\section{Brassinosteroid biosynthesis and signaling are required for the plant growth promotion by RHG1.}

Because brassinosteroids are essential phytohormones that regulate cell division, cell expansion, and plant growth (Saini et al. 2015), we wondered whether they were required for the PGP impact of RHG1. Therefore, we tested their effect on the constitutive photomorphogenesis and dwarfism (cpd) (Szekeres et al. 1996) and brassinosteroid-insensitive 1 (bril) (Jaillais et al. 2011) mutants, which are deficient in brassinosteroid biosynthesis and signaling, respectively. Because of the sterility of both the homozygous $c p d$ and bril plants, the 5-day-old seedlings were inoculated, allowing the selection of homozygous mutants from the heterozygous population. At 14 days postinoculation (DPI), the total plant fresh weight was determined, without division into shoot and root weights due to the small size of the mutants. In all four independent replicates, the fresh weights of the wild-type plants increased by 20 to $53 \%$ upon inoculation with RGH1; however, this effect was lessened in both the cpd and bril mutants, although the different replicates varied (Fig. 7A; Supplementary Fig. S7). In three of the four replicates, the fresh weights of mock-treated and RHG1inoculated cpd plants did not differ significantly, whereas, in only one replicate, the fresh weights of the RGH1-inoculated cpd plants had increased by $8 \%$, a value significantly smaller than that of $20 \%$ in the wild type (Fig. 7A). Similarly, the plant fresh weight did not increase by RHG1 inoculation in the bril mutant in three of the four replicates, except for one of the replicates, in which a smaller increase in the bril mutant than that in the wild type was observed (15 and 53\%, respectively) (Fig. 7A). These results indicate that brassinosteroid biosynthesis and signaling are necessary for the PGP effect of RHG1. Next, we analyzed whether brassinosteroid signaling was modulated by RHG1. BRI1-EMS-Suppressor 1 (BES1), a master transcription factor of brassinosteroid signaling, is phosphorylated, and inactivated in the absence of brassinosteroids. Upon brassinosteroid signaling activation, BES1 is dephosphorylated and triggered to regulate the downstream target genes (Saini et al. 2015; Yin et al. 2002). To examine the brassinosteroid signaling level upon RHG1 inoculation, the
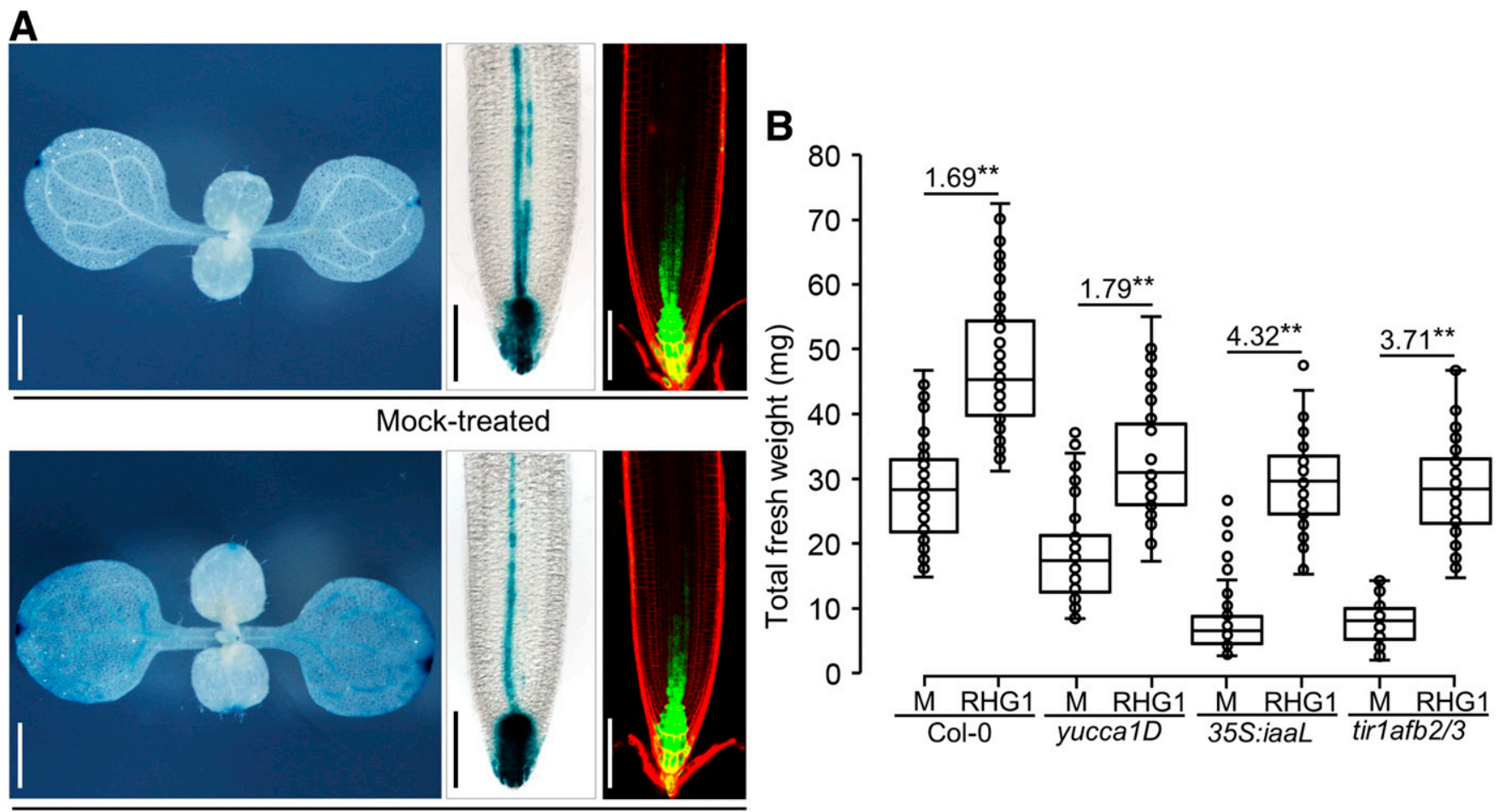

RHG1-inoculated

Fig. 5. Responses of the auxin-responsive marker lines and mutants/transgenic lines to RHG1. A, Expression of DR5: $\beta$-glucuronidase (GUS) (left and middle) and DR5: green fluorescent protein (GFP) (right) in mock-treated (top) and RHG1-inoculated (bottom) plants, respectively. Four-day-old Arabidopsis seedlings were inoculated and subjected to GUS staining or confocal microscopic imaging 4 days postinoculation. Three independent replicates were done. Bars $=1$ and $0.1 \mathrm{~mm}$ for shoot and root images, respectively. B, Effect of RHG1 on total plant fresh weight in Col-0, yuccalD, 35S:iaaL, and tirlafb2/3. Arabidopsis seeds were mock treated or inoculated with RHG1. Fresh weight was determined 18 days after initiation of germination from at least 15 plants per treatment. Results of three independent replicates were combined and shown in the plot graph. The value on top of the two boxes of each genotype is the fold change in weight between mock-treated (M) and RHG1-inoculated (RHG1) plants. Asterisks indicate significant differences between mock and inoculated plants $(* *$ indicates $P<0.01$, Student's $t$ test) 
ratio between dephosphorylated and total BES1 proteins was assessed at 4 DPI instead of 14 DPI, because the activation of the growth-triggering molecular signaling takes place before the PGP effects are observed. Additionally, the upregulation of the auxin, cytokinin, and ethylene markers was visible at 4 DPI, indicating that the interaction between the plants and the bacteria had been established at this time point. The dephosphorylation status of the BES1 proteins did not differ significantly between the mock-treated and RHG1-inoclulated plants (Fig. 7B), implying that the bacterium did not directly modify the brassinosteroid signaling, although it was required for its PGP effect.

\section{DISCUSSION}

A growing list of PGPR from a wide variety of genera such as Azospirillum, Bacillus, Azotobacter, Burkholderia, and
Pseudomonas has been discovered in the past decades (Gouda et al. 2018). Nevertheless, identification of new PGPR strains, especially those with novel or improved PGP functions, is still of great value, because it will expand our understanding of the functional diversity of PGPR and allow us to exploit the full potential of PGPR for an ameliorated agriculture. The genus Caulobacter, with $C$. vibroides as an important model system, has been well studied as a model organism for the regulation of the bacterial cell cycle, asymmetric cell division, and cellular differentiation (Woldemeskel and Goley 2017). Although bacteria of the Caulobacter genus have been isolated from the endosphere and rhizosphere of several plants, to our knowledge, RHG1 is the first Caulobacter strain for which the PGP function has been investigated in detail. The results from the genome comparison and the $16 \mathrm{~S}$ rRNA gene sequence analysis indicate that this strain might represent a new species but,

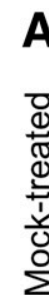
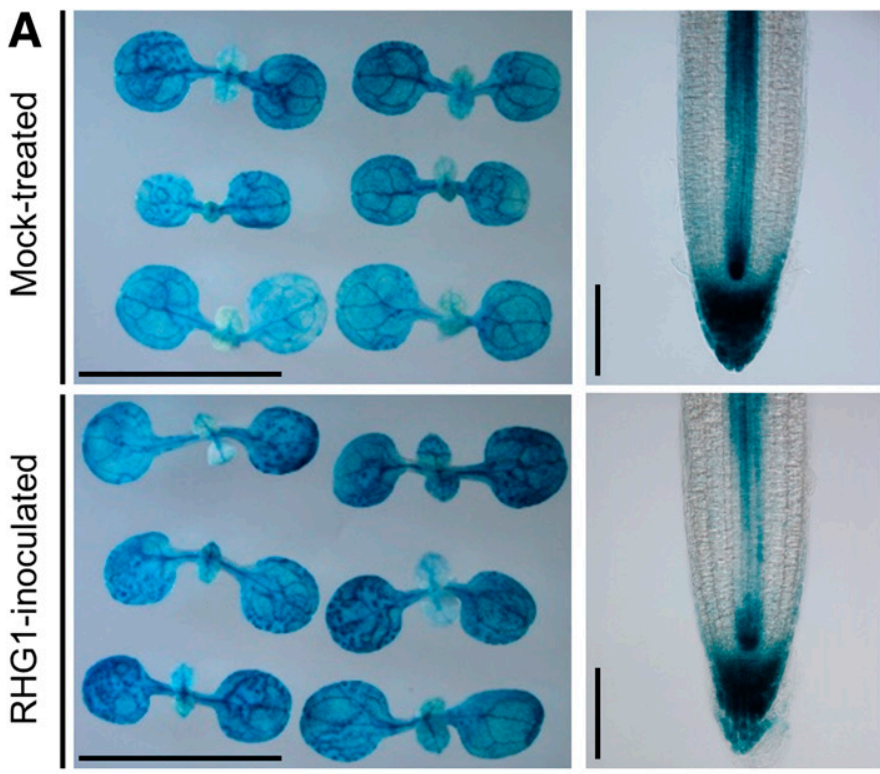

C
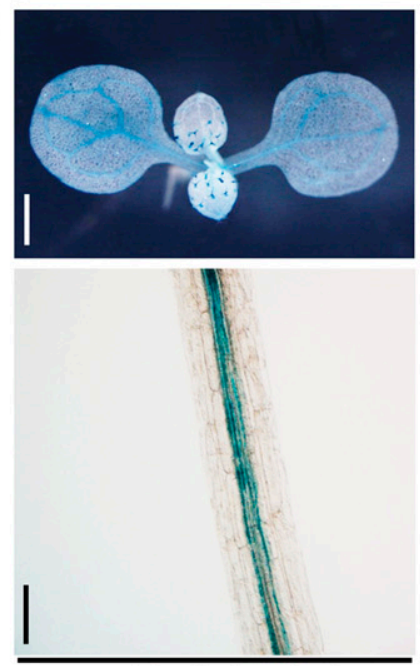

Mock-treated
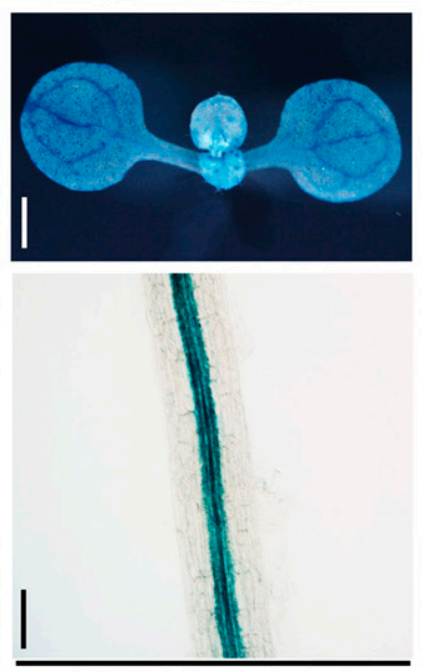

RHG1-inoculated
B

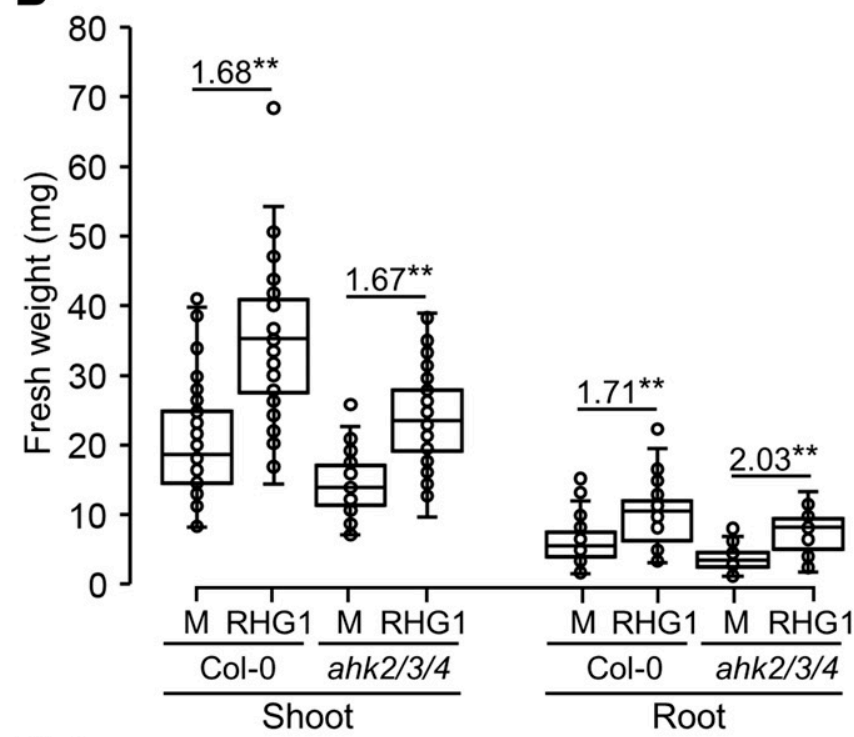

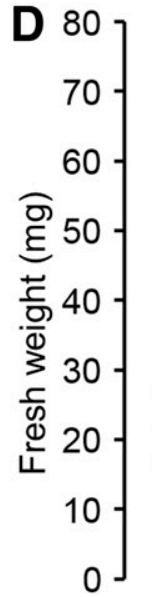
$\frac{1.76^{* *}}{\circ}$

$\frac{1.82^{* *}}{\circ}$
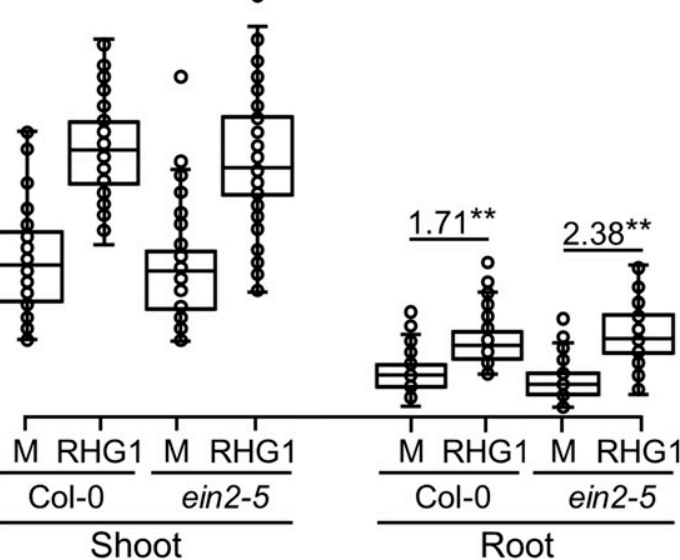

Fig. 6. Response of cytokinin- and ethylene-responsive marker lines and mutants to RHG1. A and C, Expression of $p A R R 5$ : $\beta$-glucuronidase (GUS) and $p E B S$ : GUS in mock-treated and RHG1-inoculated plants, respectively. Plants were inoculated 4 days after initiation of germination (DAIG) and subjected to GUS staining 4 days postinoculation. For pARR5:GUS (A), representative pictures of all six plants grown in one plate for mock treatment or RHG1 inoculation are shown, because of the variation in GUS staining between the plants. Three independent replicates were performed. Bars $=5 \mathrm{~mm}(\mathrm{~A})$ and $1 \mathrm{~mm}(\mathrm{C})$ for shoot and $0.1 \mathrm{~mm}$ for root images. B and D, Effect of RHG1 on Arabidopsis shoot and root fresh weights, respectively, in Col-0, ein2-5, and ahk234. Seed were mock treated or inoculated with RHG1. Fresh weight was determined at 18 DAIG from at least 20 plants per treatment. Results of four independent replicates were combined and shown in the plot graphs. The value on top of the two boxes of each genotype is the fold change in weight between mock-treated (M) and RHG1inoculated (RHG1) plants. Asterisks indicate significant differences between mock and inoculated plants (** indicates $P<0.01$, Student's $t$ test). 
because only one single strain is available, we cannot propose a formal description yet. In Arabidopsis, the RHG1 inoculation promoted the growth of both shoots and roots, with a leaf number, leaf size, shoot weight, lateral root number, total root length, and root weight higher in the inoculated plants than those in the mock control plants. Cellular analysis revealed that the RHG1 inoculation increased the number of leaf epidermal cells but decreased the cell area, suggesting that RHG1 enhances plant growth by promoting cell division. It is unclear whether the PGP factor is initially perceived by the shoot or the root or both because, in Arabidopsis, both are colonized by RHG1.

It is fascinating to uncover the mechanisms that mediate the PGP function of RHG1. Because the mock-treated plants did not benefit from the bacteria applied on the inoculated plants in the cocultivation setup, the PGP effect of RHG1 is probably not caused by easily diffusible volatiles that cause plant growth promotion by other PGPR (Kanchiswamy et al. 2015). The presence of an nirK gene in the genome of RHG1 prompted us to test whether the nirK-mediated NO production contributed to the PGP function of RHG1. However, the PGP effect by RHG1 was not reduced by the nirK gene deletion, suggesting that nirK is not crucial, in contrast to A. brasilense Sp245 (Creus et al. 2005). Hence, although both bacteria enhance the lateral root density, they seem to function through different mechanisms.

Modulation of the plant hormone landscape is another manner by which PGPR increase the root and shoot biomasses (Tsukanova et al. 2017). The bacterial genome analysis revealed that RHG1 probably lacks genes encoding proteins involved in the bacterial production of auxins, cytokinins, or ACC deaminase, all well-known PGP markers. Nevertheless, the expression of the auxin-responsive marker DR5:GUS and the cytokinin-responsive marker $p A R R 5: G U S$ was slightly upregulated in the shoot upon inoculation with RHG1. In addition to the bacterial production of these hormones, some bacteria are known to regulate the plant hormone homeostasis by affecting the expression of plant genes involved in hormone biosynthesis or transport. For example, Bacillus sp. LZR216 induces the expression of the auxin biosynthesis genes NIT1, $T A A 1$, and $Y U C C A 1$ and decreases the expression of the auxin transporters AUX1, PIN1, PIN2, and PIN3 in Arabidopsis (Wang et al. 2015), whereas Bacillus subtilis SYST2 enhances the expression of the cytokinin biosynthesis gene SICKXI in tomato plants (Tahir et al. 2017). However, given that the RHG1-mediated increase in plant weights was not attenuated in the yuccalD, 35S:iaaL, tirlafb2/3, and ahk2/3/4 mutants, the observed increase in marker gene expression is possibly indirectly caused by changes in other pathways rather than by the early perception of the bacteria. Interestingly, the upregulation of the DR5 expression in response to RHG1 inoculation occurred in shoots but not in roots, in contrast to $P$. simiae WCS417 that strongly activated the expression of DR5:YFP in roots and depended on auxin signaling for its PGP activity (Zamioudis et al. 2013). Hence, auxin might play a different role during the plant interactions with these two bacterial strains. Ethylene is a key regulator of the plant immune system and plays a crucial role in the regulation of the rhizobacteria mediated by PGPR, including P. simiae WCS417 (Pieterse et al. 1998, 2014). The expression of the ethylene-responsive marker $p E B S: G U S$ was enhanced in both shoots and roots by RHG1. Thus, it would be interesting to test whether RHG1 might have an ISR effect when the host plants are challenged with pathogens.

What could the PGP factor of RHG1 be? The RHG1mediated increase in fresh weight is dramatically lower in the brassinosteroid biosynthesis mutant $c p d$ and the signaling mutant bril than that in the wild-type plant, implying that the
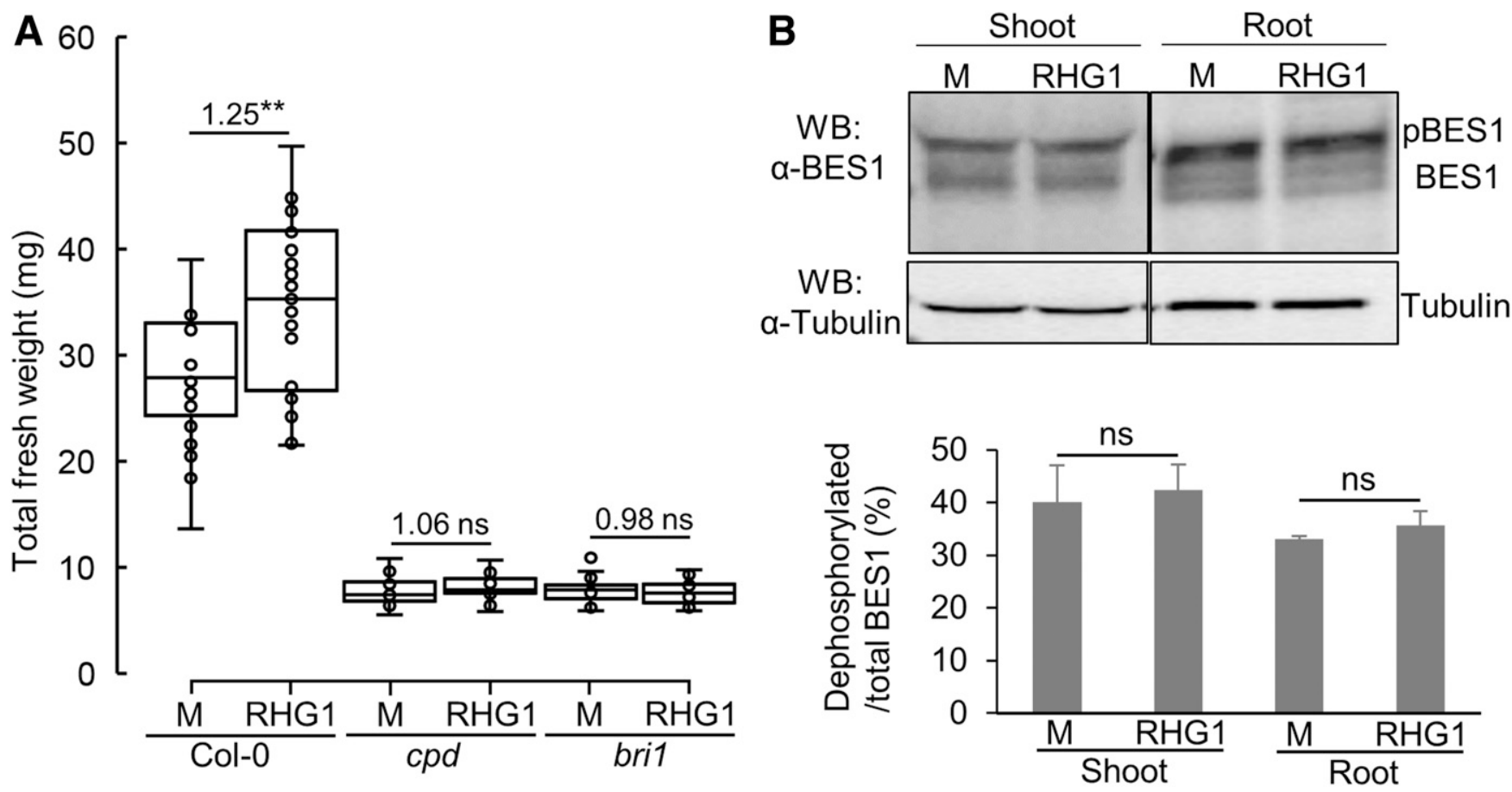

Fig. 7. Role of the brassinosteroid pathway in the plant-RHG1 interaction. A, Effect of RHG1 on plant fresh weight in Col-0, cpd, and bril. Five-day-old seedlings were mock-treated (M) or inoculated with RHG1 (RHG1). The total fresh plant weight was determined 14 days postinoculation (DPI). Four independent repeats were performed $(n \geq 15)$. The result of one replicate is shown. The value on top of the two boxes of each genotype is the fold change in weight between mock-treated (M) and RHG1-inoculated (RHG1) plants. Asterisks indicate significant differences between mock and inoculated plants (** indicates $P<0.01$ and ns indicates $P>0.05$, Student's $t$ test). B, Western blot analysis of BES1 dephosphorylation. Proteins were extracted from mocktreated (M) or RHG1-inoculated (RHG1) shoots or roots of wild-type Col-0 plants at 4 DPI, followed by Western blot with the antibody against BES1. Protein inputs were equilibrated with the antibody against tubulin. The percentage of dephosphorylated BES1 relative to the total BES1 from three biological replicates is shown; ns indicates $P>0.05$, Student's $t$ test. 
brassinosteroid pathway is required for the PGP effect. Nevertheless, the fact that the dephosphorylated level of the BES1 protein is not altered by RHG1 at 4 DPI suggests that the brassinosteroid signaling is not modified by RHG1 but could possibly be temporarily activated by RHG1, while remaining undetected at the time point of the experiment. Hence, it will be valuable to further pinpoint how the RHG1 treatment and the brassinosteroids are interconnected.

Thus, the molecular mechanism underlying the PGP function of RHG1 is notwithstanding an intriguing question, because the RHG1-produced PGP factors that are perceived by plants and induce plant growth are still unknown. In this study, we ruled out the possibility of bacterially produced auxins, cytokinins, ACC deaminase, and NO as PGP factors but we did not find any other genes or pathways encoding potential PGP factors in the draft genome of RHG1. Therefore, the PGP traits of RHG1 might be mediated by novel genes and mechanisms, because we are only just starting to comprehend the biology of soil microorganism communities and are still far away from understanding all of their gene functions. For instance, 30 to $50 \%$ of the gene sequences available in public databases still lack functional annotations as of 2017 (Sévin et al. 2017). Therefore, it is of high interest to investigate the mechanism underlying the PGP function of RHG1 in the future. For this purpose, forward genetic in planta screens for the identification of PGP traits of RHG1 will be applied.

\section{MATERIALS AND METHODS}

\section{Plant materials and bacterial strains.}

Arabidopsis thaliana (L.) Heynh. accession Col-0 and mutant and transgenic lines in the Col- 0 background were used. Mutant and transgenic lines have been described previously: DR5:GUS (Ulmasov et al. 1997), DR5:GFP (Benková et al. 2003), yuccalD (Zhao et al. 2001), 35S:iaaL (Jensen et al. 1998), tirlafb2/3 (Dharmasiri et al. 2005), pARR5:GUS (D'Agostino et al. 2000), ahk2/3/4 (Nishimura et al. 2004), pEBS:GUS (Stepanova et al. 2007), ein2-5 (Wang et al. 2007), bril (Jaillais et al. 2011), and cpd (Szekeres et al. 1996).

The Caulobacter sp. strain RHG1 was isolated from surfacesterilized roots of the maize variety LG30270 grown in agricultural field soil $\left(50^{\circ} 58^{\prime} 41^{\prime \prime} \mathrm{N}, 3^{\circ} 46^{\prime} 47.28^{\prime \prime} \mathrm{E}\right.$; Merelbeke, Belgium). The GFP-labeled strain RHG1::GFP and the nirK gene deletion strain RHG1 $\Delta$ nirK were constructed in this study.

\section{Plant inoculation and growth conditions.}

Caulobacter strains, including the wild-type strain RHG1, the labeled strain RHG1::GFP, and the mutant strain RHG1 $\Delta$ nirK, were routinely grown in R2A medium (Reasoner and Geldreich 1985) at $28^{\circ} \mathrm{C}$. The bacterial inoculum was made by subculturing $1 \mathrm{ml}$ of overnight culture in $15 \mathrm{ml}$ of fresh R2A medium for $3 \mathrm{~h}$. Cells were collected by centrifugation for 5 min at 3,000 $\times g$, washed once, and resuspended in phosphatebuffered saline (PBS) solution. The bacterial concentration was adjusted to an optical density at $600 \mathrm{~nm}=0.1\left(10^{7} \mathrm{CFU} / \mathrm{ml}\right)$ and further diluted 10- or 1,000-fold for seedling or seed inoculation, respectively. For seed inoculation, seeds were surface sterilized with chlorine gas and five seeds were sown per square Petri plate $(120$ by $120 \mathrm{~mm})$ with half-strength Murashige and Skoog (MS) medium and 0.8\% (wt/vol) agar. Subsequently, $1 \mu \mathrm{l}$ of PBS solution or bacterial inoculum was applied on each seed for mock treatment or bacterial inoculation, whereafter the plates were kept at $4^{\circ} \mathrm{C}$ in the dark for 2 days to synchronize seed germination, then placed vertically in the growth chamber. For seedling inoculation, seeds were sown and allowed to germinate under the same conditions.
Four-day-old seedlings (or otherwise specified) were transferred to fresh half-strength MS agar plates and inoculated by pipetting $8 \mu \mathrm{l}$ of PBS solution or bacterial inoculum onto the shoot and root of each seedling. After inoculation, all plants were grown vertically in a growth chamber at $21^{\circ} \mathrm{C}$ with a photoperiod of $16 \mathrm{~h}$ of light and $8 \mathrm{~h}$ of darkness.

\section{Measurement of the root architecture and plant weight parameters.}

For the measurement of the root architecture parameters, images of Petri plates with Arabidopsis seedlings were captured by means of an EPSON Expression 11000XL A3 scanner. The lateral root number was counted manually. The root length was measured with the NeuronJ plugin of the ImageJ software. The fresh shoot and root masses were weighed by separating the seedlings at the shoot-root junction. Excised shoots and roots were quickly cleaned with paper tissues to remove the surface water and bacteria and immediately weighed on an analytical balance with a $0.1-\mathrm{mg}$ resolution.

\section{Enumeration of bacterial colonization.}

Arabidopsis seeds were inoculated with RHG1 in agar plates as described above. To determine the RHG1 population on the shoots and roots, 10 shoots or roots were removed from the plates, pooled, and weighed at 10 DAIG, whereafter the samples were transferred to a sterile mortar and ground in $1 \mathrm{ml}$ of PBS solution, followed by successive 10-fold PBS dilutions from $10^{-1}$ to $10^{-8}$. The diluted extract $\left(100 \mu \mathrm{l}\right.$ at $10^{-6}, 10^{-7}$, and $10^{-8}$ dilutions) was plated on a R2A medium plate. Three plates were plated for each dilution. The CFU per milligram of $\mathrm{FW}$ was determined after 3 days of incubation at $28^{\circ} \mathrm{C}$. Three independent biological replicates were done.

\section{Leaf cellular analysis.}

Four-day-old Arabidopsis seedlings were inoculated with RHG1. At 14 DPI, the total leaf blade area of cleared leaves was measured for both the first and second leaf of 15 plants per treatment under a dark-field binocular microscope. For three representative plants per treatment, the abaxial epidermal cells in the middle of the leaves were drawn with a microscope equipped with differential interference contrast optics (DM LB; Leica) and a drawing tube. Photographs of leaves and scanned cell drawings were used to measure leaf and cell areas, as described previously (Andriankaja et al. 2012).

\section{GFP labeling of RHG1.}

The mini-Tn5-based transposon delivery plasmid pUTgfp $2 x$ (Tombolini et al. 1997) was used for chromosomal insertion of the marker gene. The plasmid was introduced into RHG1 by triparental conjugation. The recipient strain RHG1 was grown overnight in PYE medium $(0.2 \%$ [wt/vol] Bacto peptone, $0.1 \%$ [wt/vol] yeast extract, $1 \mathrm{mM} \mathrm{MgSO}_{4}$, and $0.5 \mathrm{mM} \mathrm{CaCl}_{2}$ ) and back diluted 1:15 into fresh medium, followed by a 5-h incubation at $28^{\circ} \mathrm{C}$. In parallel, donor strain Escherichia coli with the plasmid pUTgfp2x and helper strain E. coli pRK2013 were grown overnight in lysogeny broth medium and back diluted 1: 100 into fresh medium, followed by a 5 -h incubation at $37^{\circ} \mathrm{C}$. The resulting cultures were combined in a recipient/donor/ helper ratio of $8: 1: 1$, collected by centrifugation for $1 \mathrm{~min}$ at $5,000 \times g$, and then grown on PYE agar plates without antibiotics to allow overnight conjugation. Transconjugants were selected on PYE agar supplemented with kanamycin at $50 \mu \mathrm{g} / \mathrm{ml}$ to select for the RHG1 strain with genomic integration of the marker gene and nalidixic acid at $20 \mu \mathrm{g} / \mathrm{ml}$ to counterselect against the E. coli strains. Colonies with a green fluorescent phenotype were selected and further confirmed by PCR amplification of the GFP gene and by sequencing of the $16 \mathrm{~S}$ gene. 
Confocal microscopic analysis of colonization.

Arabidopsis seeds were inoculated with the GFP-tagged strain RHG1::GFP as described above. Fourteen-day-old plants were examined for colonization by means of live tissue or cleared tissue imaging. For live tissue imaging, plant samples were gently washed with half-strength MS medium, then mounted on microscope slides, and processed with an Olympus FluoView 1000 confocal microscope. For cleared tissue imaging, plants were fixed and cleared according to the ClearSee protocol described previously (Kurihara et al. 2015). Briefly, plants were fixed with $4 \%$ (wt/vol) paraformaldehyde in PBS solution for $1 \mathrm{~h}$ with vacuum treatment, whereafter they were rinsed twice with PBS solution. Subsequently, fixed samples were cleared in ClearSee solution $(10 \%$ [wt/vol] xylitol, $15 \%$ [wt/vol] sodium deoxycholate, and $25 \%$ [wt/vol] urea in water) for 2 weeks at room temperature. Cleared samples were analyzed with a Zeiss LSM5 710 confocal microscope.

\section{Genome sequencing, assembly, and annotation.}

The Caulobacter sp. RHG1 genome was sequenced with an Illumina HiSeq 4000 platform (PE150 reads) at the Oxford Genomics Center. The quality of the reads was evaluated by means of the FastQC v0.11.8 software (Schmieder and Edwards 2011). The assembly of the genome was achieved through the Shovill pipeline that mainly applies SPAdes v3.12.0 (Bankevich et al. 2012). In addition, the Timmomatic v0.38 option in the Shovill pipeline was included into the command line to implement the trimming of the reads. The quality of the assembly was assessed with the software Quast v4.6.3 (Gurevich et al. 2013) and contigs smaller than 1,000 bp were removed. The genome was annotated with the Rapid Annotation using Subsystem Technology (Overbeek et al. 2014) and Prokka software (Seemann 2014). BLAST v2.6.0 (Camacho et al. 2009) was used to verify the absence of the genes involved in plant growth promotion that were not present in the genome annotation of RHG1. The Caulobacter sp. RHG1 genome sequence data were deposited in the GenBank under genome accession number SMZP00000000.

\section{Phylogenomic analysis.}

For the phylogenomic analysis of Caulobacter sp. RHG1, the genomes of 38 strains previously classified within the genus Caulobacter and 3 strains from the genus Brevundimonas, used as outgroup, were downloaded from the NCBI database. Initially, all available genomes of the Caulobacter strains in the NCBI database were included into the analysis but those that lacked several essential core genes were removed to avoid possible bias created by the presence of incomplete genomes. The bcgTree pipeline (Ankenbrand and Keller 2016) was used to compare 107 essential bacterial core genes of the 42 strains. The comparison was done by performing 1,000 bootstraps. Additionally, this pipeline generated a phylogenomic tree with RaxML that was visualized with interactive Tree of Life v4.2.3 (Letunic and Bork 2016). Moreover, all strains were compared with the OrthoANIu (Yoon et al. 2017) method for their similarity determination.

\section{Phylogenetic analysis.}

Because not all of the genome sequences were available for all 11 valid Caulobacter spp., a maximum-likelihood tree was constructed based on the available sequences of the 16S rRNA genes with the MEGA v10.0.5 software (Kumar et al. 2008).

\section{nirK gene deletion strain construction.}

The nirK gene deletion was achieved through a recombinationbased method with the allelic exchange vector pNPTS138 that contains the kanamycin resistance cassette and the sucrose-sensitive counterselection marker sacB (Ried and Collmer 1987). To this end, a fused PCR product containing the 500-bp upstream region (amplified with the primers nirk_upstream_forward: aactg cagctcttccatgcaggcgatcg and nirk_upstream_reverse: gttgtagaca agattgatatctagatacacgttcetatccecgggcgec) and the 500-bp downstream region (amplified with the primers nirk_downstream_forward: gtgtatctagatatcaatcttgtctacaaccctcgaaacccegg ggcgg and nirk_downstream_reverse: ccggaattcgctcgttctgccgcc aggccgag) of the nirK gene was cloned into the pNPTS138 vector. The resulting plasmid was introduced into RHG1 by conjugation through the $E$. coli strain S17-1 with kanamycin to select for the presence of the integrated plasmid DNA and with nalidixic acid to counterselect against $E$. coli. Colonies were further confirmed by PCR for plasmid DNA integration and then grown in PYE medium overnight under nonselective conditions to allow a second recombination. Thereafter, cells were selected on PYE medium supplemented with 5\% (wt/vol) sucrose and further tested for loss of kanamycin resistance. Sucrose-resistant and kanamycin-sensitive clones were further confirmed by PCR for the deletion of the nirK gene.

\section{Western blot analysis of BES1 dephosphorylation.}

Four-day-old wild-type Arabidopsis seedlings were mock treated or inoculated with RHG1. Shoot and root samples separated from seedlings at 4 DPI were homogenized in liquid nitrogen. Total proteins were extracted with a buffer containing $20 \mathrm{mM}$ Tris $\cdot \mathrm{HCl}$ (pH 7.5), $150 \mathrm{mM} \mathrm{NaCl}, 1 \%$ (wt/vol) sodium dodecyl sulfate, $100 \mathrm{mM}$ dithiothreitol, and EDTAfree Protease Inhibitor Cocktail (Roche). For protein detection, the antibodies polyclonal $\alpha$-BES1 (Yin et al. 2002), $1 / 4,000$ dilution, and monoclonal $\alpha$-Tubulin (SigmaAldrich), 1/10,000 dilution, were used. For the BES1 dephosphorylation assay, the ratio of the dephosphorylated BES1 to the total BES1 proteins was quantified based on the signal intensity. The loading was adjusted to an equal level based on the amount of tubulin. The signal intensity was determined with the Image Lab software.

\section{GUS histochemical staining.}

Four-day-old seedlings of the DR5:GUS, pARR5:GUS, and $p E B S: G U S$ reporter lines were mock treated or inoculated with Caulobacter sp. RHG1 and subjected to GUS histochemical staining at 4 DPI. Plants were incubated in GUS staining solution (100 mM Tris [pH 7], $50 \mathrm{mM} \mathrm{NaCl}, 1 \mathrm{mM} \mathrm{K}_{3}\left[\mathrm{Fe}(\mathrm{CN})_{6}\right.$ ], and $2 \mathrm{mM}$ 5-bromo-4-chloro-3-indolyl $\beta$-D-glucuronide cyclohexylammonium salt) overnight at $37^{\circ} \mathrm{C}$, followed by decoloring in $70 \%$ (vol/ $\mathrm{vol}$ ) ethanol. Plant shoot or root samples were examined under a stereomicroscope (Leica MZ16) or a differential interference contrast microscope (Olympus BX51), respectively.

\section{Fluorescence microscopy.}

For detection of the GFP signal in the DR5:GFP reporter line, 4-day-old seedlings were mock treated or inoculated with Caulobacter sp. RHG1, followed by microscopic analysis at 4 DPI. Seedlings were stained with propidium iodide (PI) at $10 \mu \mathrm{g} / \mathrm{ml}$ for $1 \mathrm{~min}$ and observed under an Olympus FluoView 1000 confocal microscope. The excitation wavelength was $488 \mathrm{~nm}$ for GFP and $559 \mathrm{~nm}$ for PI. Emission was detected at 500 to $530 \mathrm{~nm}$ for GFP and 580 to $619 \mathrm{~nm}$ for PI.

\section{ACKNOWLEDGMENTS}

We thank B. Mitter and S. Compant (Austrian Institute of Technology, Vienna, Austria) and P. H. Viollier (University of Geneva, Switzerland) for kindly providing the plasmid pUTgfp $2 x$ and pNPTS138, respectively; A. Baekelandt for cellular analysis; and M. De Cock for help in preparing the manuscript. 


\section{AUTHOR-RECOMMENDED INTERNET RESOURCES}

GenBank: https://www.ncbi.nlm.nih.gov/genbank/

NCBI database: https://www.ncbi.nlm.nih.gov/

\section{LITERATURE CITED}

Agler, M. T., Ruhe, J., Kroll, S., Morhenn, C., Kim, S.-T., Weigel, D., and Kemen, E. M. 2016. Microbial hub taxa link host and abiotic factors to plant microbiome variation. PLoS Biol. 14:e1002352.

Andriankaja, M., Dhondt, S., De Bodt, S., Vanhaeren, H., Coppens, F., De Milde, L., Mühlenbock, P., Skirycz, A., Gonzalez, N., Beemster, G. T. S., and Inzé, D. 2012. Exit from proliferation during leaf development in Arabidopsis thaliana: A not-so-gradual process. Dev. Cell 22:64-78.

Ankenbrand, M. J., and Keller, A. 2016. bcgTree: Automatized phylogenetic tree building from bacterial core genomes. Genome 59:783-791.

Bai, Y., Müller, D. B., Srinivas, G., Garrido-Oter, R., Potthoff, E., Rott, M., Dombrowski, N., Münch, P. C., Spaepen, S., Remus-Emsermann, M., Hüttel, B., McHardy, A. C., Vorholt, J. A., and Schulze-Lefert, P. 2015. Functional overlap of the Arabidopsis leaf and root microbiota. Nature 528:364-369.

Bankevich, A., Nurk, S., Antipov, D., Gurevich, A. A., Dvorkin, M., Kulikov, A. S., Lesin, V. M., Nikolenko, S. I., Pham, S., Prjibelski, A. D., Pyshkin, A. V., Sirotkin, A. V., Vyahhi, N., Tesler, G., Alekseyev, M. A., and Pevzner, P. A. 2012. SPAdes: A new genome assembly algorithm and its applications to single-cell sequencing. J. Comput. Biol. 19: 455-477.

Benková, E., Michniewicz, M., Sauer, M., Teichmann, T., Seifertová, D., Jürgens, G., and Friml, J. 2003. Local, efflux-dependent auxin gradients as a common module for plant organ formation. Cell 115 : 591-602.

Berendsen, R. L., van Verk, M. C., Stringlis, I. A., Zamioudis, C., Tommassen, J., Pieterse, C. M. J., and Bakker, P. A. H. M. 2015. Unearthing the genomes of plant-beneficial Pseudomonas model strains WCS358, WCS374 and WCS417. BMC Genomics 16:539.

Berendsen, R. L., Vismans, G., Yu, K., Song, Y., de Jonge, R., Burgman, W. P., Burmølle, M., Herschend, J., Bakker, P. A. H. M., and Pieterse, C. M. J. 2018. Disease-induced assemblage of a plant-beneficial bacterial consortium. ISME J. 12:1496-1507.

Bhattacharyya, D., Garladinne, M., and Lee, Y. H. 2015. Volatile indole produced by rhizobacterium Proteus vulgaris JBLS202 stimulates growth of Arabidopsis thaliana through auxin, cytokinin, and brassinosteroid pathways. J. Plant Growth Regul. 34:158-168.

Camacho, C., Coulouris, G., Avagyan, V., Ma, N., Papadopoulos, J., Bealer, K., and Madden, T. L. 2009. BLAST+: Architecture and applications. BMC Bioinformatics 10:421.

Creus, C. M., Graziano, M., Casanovas, E. M., Pereyra, M. A., Simontacchi, M., Puntarulo, S., Barassi, C. A., and Lamattina, L. 2005. Nitric oxide is involved in the Azospirillum brasilense-induced lateral root formation in tomato. Planta 221:297-303.

D'Agostino, I. B., Deruère, J., and Kieber, J. J. 2000. Characterization of the response of the Arabidopsis response regulator gene family to cytokinin. Plant Physiol. 124:1706-1717.

Dharmasiri, N., Dharmasiri, S., Weijers, D., Lechner, E., Yamada, M., Hobbie, L., Ehrismann, J. S., Jürgens, G., and Estelle, M. 2005. Plant development is regulated by a family of auxin receptor $\mathrm{F}$ box proteins. Dev. Cell 9:109-119.

Edulamudi, P., Antony Masilamani, A. J., Divi, V. R. S. G., and Konada, V. M. 2011. Novel root nodule bacteria belonging to the genus Caulobacter. Lett. Appl. Microbiol. 53:587-591.

Fitzpatrick, C. R., Copeland, J., Wang, P. W., Guttman, D. S., Kotanen, P. M., and Johnson, M. T. J. 2018. Assembly and ecological function of the root microbiome across angiosperm plant species. Proc. Natl. Acad. Sci. U.S.A. 115:E1157-E1165.

Glick, B. R. 2014. Bacteria with ACC deaminase can promote plant growth and help to feed the world. Microbiol. Res. 169:30-39.

Gouda, S., Kerry, R. G., Das, G., Paramithiotis, S., Shin, H.-S., and Patra, J. K. 2018. Revitalization of plant growth promoting rhizobacteria for sustainable development in agriculture. Microbiol. Res. 206:131-140.

Gurevich, A., Saveliev, V., Vyahhi, N., and Tesler, G. 2013. QUAST: Quality assessment tool for genome assemblies. Bioinformatics 29: 1072-1075

Hontzeas, N., Zoidakis, J., Glick, B. R., and Abu-Omar, M. M. 2004 Expression and characterization of 1-aminocyclopropane-1-carboxylate deaminase from the rhizobacterium Pseudomonas putida UW4: A key enzyme in bacterial plant growth promotion. Biochim. Biophys. Acta 1703:11-19.
Jaillais, Y., Belkhadir, Y., Balsemão-Pires, E., Dangl, J. L., and Chory, J. 2011. Extracellular leucine-rich repeats as a platform for receptor/coreceptor complex formation. Proc. Natl. Acad. Sci. U.S.A. 108:85038507.

Jensen, P. J., Hangarter, R. P., and Estelle, M. 1998. Auxin transport is required for hypocotyl elongation in light-grown but not dark-grown Arabidopsis. Plant Physiol. 116:455-462.

Kalve, S., De Vos, D., and Beemster, G. T. S. 2014. Leaf development: A cellular perspective. Front. Plant Sci. 5:362.

Kanchiswamy, C. N., Malnoy, M., and Maffei, M. E. 2015. Bioprospecting bacterial and fungal volatiles for sustainable agriculture. Trends Plant Sci. 20:206-211.

Kumar, S., Nei, M., Dudley, J., and Tamura, K. 2008. MEGA: A biologistcentric software for evolutionary analysis of DNA and protein sequences. Brief Bioinform. 9:299-306.

Kurihara, D., Mizuta, Y., Sato, Y., and Higashiyama, T. 2015. ClearSee: A rapid optical clearing reagent for whole-plant fluorescence imaging. Development 142:4168-4179.

Kwak, M.-J., Kong, H. G., Choi, K., Kwon, S.-K., Song, J. Y., Lee, J., Lee, P. A., Choi, S. Y., Seo, M., Lee, H. J., Jung, E. J., Park, H., Roy, N., Kim, H., Lee, M. M., Rubin, E. M., Lee, S.-W., and Kim, J. F. 2018 Rhizosphere microbiome structure alters to enable wilt resistance in tomato. Nat. Biotechnol. 36:1100-1109. [Author correction: Nat. Biotechnol. 36:1117].

Lemanceau, P., Blouin, M., Muller, D., and Moënne-Loccoz, Y. 2017. Let the core microbiota be functional. Trends Plant Sci. 22:583-595.

Letunic, I., and Bork, P. 2016. Interactive tree of life (iTOL) v3: An online tool for the display and annotation of phylogenetic and other trees. Nucleic Acids Res. 44:W242-W245.

Liu, F., Xing, S., Ma, H., Du, Z., and Ma, B. 2013. Cytokinin-producing, plant growth-promoting rhizobacteria that confer resistance to drought stress in Platycladus orientalis container seedlings. Appl. Microbiol. Biotechnol. 97:9155-9164.

Mitter, B., Petric, A., Shin, M. W., Chain, P. S. G., Hauberg-Lotte, L., Reinhold-Hurek, B., Nowak, J., and Sessitsch, A. 2013. Comparative genome analysis of Burkholderia phytofirmans PsJN reveals a wide spectrum of endophytic lifestyles based on interaction strategies with host plants. Front. Plant Sci. 4:120.

Moya, G., Yan, Z.-F., Won, K., Yang, J.-E., Wang, Q.-J., Kook, M., and Yi, T.-H. 2017. Caulobacter hibisci sp. nov., isolated from rhizosphere of Hibiscus syriacus L. (Mugunghwa flower). Int. J. Syst. Evol. Microbiol. 67:3167-3173.

Nishimura, C., Ohashi, Y., Sato, S., Kato, T., Tabata, S., and Ueguchi, C. 2004. Histidine kinase homologs that act as cytokinin receptors possess overlapping functions in the regulation of shoot and root growth in Arabidopsis. Plant Cell 16:1365-1377.

Overbeek, R., Olson, R., Pusch, G. D., Olsen, G. J., Davis, J. J., Disz, T., Edwards, R. A., Gerdes, S., Parrello, B., Shukla, M., Vonstein, V., Wattam, A. R., Xia, F., and Stevens, R. 2014. The SEED and the Rapid Annotation of microbial genomes using Subsystems Technology (RAST). Nucleic Acids Res. 42:D206-D214.

Pallai, R., Hynes, R. K., Verma, B., and Nelson, L. M. 2012. Phytohormone production and colonization of canola (Brassica napus L.) roots by Pseudomonas fluorescens 6-8 under gnotobiotic conditions. Can. J. Microbiol. 58:170-178.

Pieterse, C. M. J., van Wees, S. C. M., van Pelt, J. A., Knoester, M., Laan, R., Gerrits, H., Weisbeek, P. J., and van Loon, L. C. 1998. A novel signaling pathway controlling induced systemic resistance in Arabidopsis. Plant Cell 10:1571-1580.

Pieterse, C. M. J., Zamioudis, C., Berendsen, R. L., Weller, D. M., Van Wees, S. C. M., and Bakker, P. A. H. M. 2014. Induced systemic resistance by beneficial microbes. Annu. Rev. Phytopathol. 52:347-375.

Pothier, J. F., Prigent-Combaret, C., Haurat, J., Moënne-Loccoz, Y., and Wisniewski-Dyé, F. 2008. Duplication of plasmid-borne nitrite reductase gene nirK in the wheat-associated plant growth-promoting rhizobacterium Azospirillum brasilense Sp245. Mol. Plant-Microbe Interact. 21:831-842.

Reasoner, D. J., and Geldreich, E. E. 1985. A new medium for the enumeration and subculture of bacteria from potable water. Appl. Environ. Microbiol. 49:1-7.

Richardson, A. E., Barea, J.-M., McNeill, A. M., and Prigent-Combaret, C. 2009. Acquisition of phosphorus and nitrogen in the rhizosphere and plant growth promotion by microorganisms. Plant Soil 321:305-339.

Ried, J. L., and Collmer, A. 1987. An nptI-sacB-sacR cartridge for constructing directed, unmarked mutations in gram-negative bacteria by marker exchange-eviction mutagenesis. Gene 57:239-246.

Saini, S., Sharma, I., and Pati, P. K. 2015. Versatile roles of brassinosteroid in plants in the context of its homoeostasis, signaling and crosstalks. Front. Plant Sci. 6:950. 
Sami, F., Faizan, M., Faraz, A., Siddiqui, H., Yusuf, M., and Hayat, S. 2018. Nitric oxide-mediated integrative alterations in plant metabolism to confer abiotic stress tolerance, NO crosstalk with phytohormones and NO-mediated post translational modifications in modulating diverse plant stress. Nitric Oxide 73:22-38.

Schaller, G. E., Street, I. H., and Kieber, J. J. 2014. Cytokinin and the cell cycle. Curr. Opin. Plant Biol. 21:7-15.

Schmieder, R., and Edwards, R. 2011. Quality control and preprocessing of metagenomic datasets. Bioinformatics 27:863-864.

Seemann, T. 2014. Prokka: Rapid prokaryotic genome annotation. Bioinformatics 30:2068-2069.

Sévin, D. C., Fuhrer, T., Zamboni, N., and Sauer, U. 2017. Nontargeted in vitro metabolomics for high-throughput identification of novel enzymes in Escherichia coli. Nat. Methods 14:187-194.

Spaepen, S., Bossuyt, S., Engelen, K., Marchal, K., and Vanderleyden, J. 2014. Phenotypical and molecular responses of Arabidopsis thaliana roots as a result of inoculation with the auxin-producing bacterium Azospirillum brasilense. New Phytol. 201:850-861.

Stepanova, A. N., Yun, J., Likhacheva, A. V., and Alonso, J. M. 2007. Multilevel interactions between ethylene and auxin in Arabidopsis roots. Plant Cell 19:2169-2185.

Stringlis, I. A., Proietti, S., Hickman, R., Van Verk, M. C., Zamioudis, C., and Pieterse, C. M. J. 2018. Root transcriptional dynamics induced by beneficial rhizobacteria and microbial immune elicitors reveal signatures of adaptation to mutualists. Plant J. 93:166-180.

Sun, L.-N., Yang, E.-D., Hou, X.-T., Wei, J.-C., Yuan, Z.-X., and Wang, W.-Y. 2017. Caulobacter rhizosphaerae sp. nov., a stalked bacterium isolated from rhizosphere soil. Int. J. Syst. Evol. Microbiol. 67:1771-1776.

Szekeres, M., Németh, K., Koncz-Kálmán, Z., Mathur, J., Kauschmann, A., Altmann, T., Rédei, G. P., Nagy, F., Schell, J., and Koncz, C. 1996. Brassinosteroids rescue the deficiency of CYP90, a cytochrome P450, controlling cell elongation and de-etiolation in Arabidopsis. Cell 85:171-182.

Tahir, H. A. S., Gu, Q., Wu, H., Raza, W., Hanif, A., Wu, L., Colman, M. V., and Gao, X. 2017. Plant growth promotion by volatile organic compounds produced by Bacillus subtilis SYST2. Front. Microbiol. 8:171.

Toju, H., Peay, K. G., Yamamichi, M., Narisawa, K., Hiruma, K., Naito, K., Fukuda, S., Ushio, M., Nakaoka, S., Onoda, Y., Yoshida, K., Schlaeppi, K., Bai, Y., Sugiura, R., Ichihashi, Y., Minamisawa, K., and Kiers, E. T. 2018. Core microbiomes for sustainable agroecosystems. Nat. Plants 4:247-257.

Tombolini, R., Unge, A., Davey, M. E., de Bruijn, F. J., and Jansson, J. K. 1997. Flow cytometric and microscopic analysis of GFP-tagged Pseudomonas fluorescens bacteria. FEMS Microbiol. Ecol. 22:17-28.

Tsukanova, K. A., Chebotar, V. K., Meyer, J. J. M., and Bibikova, T. N. 2017. Effect of plant growth-promoting rhizobacteria on plant hormone homeostasis. S. Afr. J. Bot. 113:91-102.

Ulmasov, T., Murfett, J., Hagen, G., and Guilfoyle, T. J. 1997. Aux/IAA proteins repress expression of reporter genes containing natural and highly active synthetic auxin response elements. Plant Cell 9:1963-1971.
Vacheron, J., Desbrosses, G., Bouffaud, M.-L., Touraine, B., MoënneLoccoz, Y., Muller, D., Legendre, L., Wisniewski-Dyé, F., and PrigentCombaret, C. 2013. Plant growth-promoting rhizobacteria and root system functioning. Front. Plant Sci. 4:356.

Vacheron, J., Desbrosses, G., Renoud, S., Padilla, R., Walker, V., Muller, D., and Prigent-Combaret, C. 2018. Differential contribution of plantbeneficial functions from Pseudomonas kilonensis F113 to root system architecture alterations in Arabidopsis thaliana and Zea mays. Mol. Plant-Microbe Interact. 31:212-223.

Van Deynze, A., Zamora, P., Delaux, P.-M., Heitmann, C., Jayaraman, D., Rajasekar, S., Graham, D., Maeda, J., Gibson, D., Schwartz, K. D., Berry, A. M., Bhatnagar, S., Jospin, G., Darling, A., Jeannotte, R., Lopez, J., Weimer, B. C., Eisen, J. A., Shapiro, H.-Y., Ané, J.-M., and Bennett, A. B. 2018. Nitrogen fixation in a landrace of maize is supported by a mucilage-associated diazotrophic microbiota. PLoS Biol. 16:e2006352.

Walters, W. A., Jin, Z., Youngblut, N., Wallace, J. G., Sutter, J., Zhang, W., González-Peña, A., Peiffer, J., Koren, O., Shi, Q., Knight, R., Glavina del Rio, T., Tringe, S. G., Buckler, E. S., Dangl, J. L., and Ley, R. E. 2018 Large-scale replicated field study of maize rhizosphere identifies heritable microbes. Proc. Natl. Acad. Sci. U.S.A. 115:7368-7373.

Wang, J., Zhang, Y., Li, Y., Wang, X., Nan, W., Hu, Y., Zhang, H., Zhao, C., Wang, F., Li, P., Shi, H., and Bi, Y. 2015. Endophytic microbes Bacillus sp. LZR216-regulated root development is dependent on polar auxin transport in Arabidopsis seedlings. Plant Cell Rep. 34:1075-1087.

Wang, Y., Liu, C., Li, K., Sun, F., Hu, H., Li, X., Zhao, Y., Han, C., Zhang, W., Duan, Y., Liu, M., and Li, X. 2007. Arabidopsis EIN2 modulates stress response through abscisic acid response pathway. Plant Mol. Biol. 64:633-644.

Woldemeskel, S. A., and Goley, E. D. 2017. Shapeshifting to survive: Shape determination and regulation in Caulobacter crescentus. Trends Microbiol. 25:673-687.

Yin, Y., Wang, Z.-Y., Mora-Garcia, S., Li, J., Yoshida, S., Asami, T., and Chory, J. 2002. BES1 accumulates in the nucleus in response to brassinosteroids to regulate gene expression and promote stem elongation. Cell 109:181-191.

Yoon, S.-H., Ha, S., Lim, J., Kwon, S., and Chun, J. 2017. A large-scale evaluation of algorithms to calculate average nucleotide identity. Antonie van Leeuwenhoek 110:1281-1286.

Zamioudis, C., Mastranesti, P., Dhonukshe, P., Blilou, I., and Pieterse, C. M. J. 2013. Unraveling root developmental programs initiated by beneficial Pseudomonas spp. bacteria. Plant Physiol. 162:304-318.

Zhao, Y., Christensen, S. K., Fankhauser, C., Cashman, J. R., Cohen, J. D., Weigel, D., and Chory, J. 2001. A role for flavin monooxygenase-like enzymes in auxin biosynthesis. Science 291:306-309.

Zumft, W. G. 1997. Cell biology and molecular basis of denitrification. Microbiol. Mol. Biol. Rev. 61:533-616. 